\title{
Structural evolution of graphene quantum dots during thermal decomposition of citric acid and the corresponding photoluminescence
}

\author{
Shujun Wang ${ }^{1}$, Zhigang Chen ${ }^{2}$, Ivan Cole ${ }^{3}$ and ${\mathrm{Qin} \mathrm{Li}^{1 *}}^{*}$ \\ 1. Environmental Engineering \& Queensland Miro- and Nanotechnology Centre, Griffith University, \\ Nathan, QLD 4111, Australia
}

2. Materials Engineering, the University of Queensland, St Lucia, QLD 4072, Australia

3. CSIRO Materials Science and Engineering, Clayton VIC 3168, Australia

\begin{abstract}
The thermally decomposed citric acid (TDCA) possesses either excitation-dependent or excitationindependent fluorescence as well as different quantum yields with varying synthesis conditions (i.e. temperature and reaction duration). These photoluminescent (PL) properties were found to be mainly determined by the quantitative competition between the graphene quantum dots (average size in the range $0.7 \sim 1 \mathrm{~nm}$ ) and the large-inhomogeneously-sized particles. Thermal induced reduction of oxygen containing functionalities leads to an enhancing effect to the PL of GQDs. The study reveals the structural evolution of the GQDs upon thermal treatment and attempts to establish its relationship to the PL property. The GQDs synthesized in this study are excellent sensing materials for trivalent iron cation with both notable selectivity and sensitivity.
\end{abstract}

\section{Introduction}

Graphene sheets smaller than 100nm show quantum confinement behaviour which generates band gaps in their electronic band structures[1] in contrast to the well-known zero-gap feature of graphene sheets with much larger sizes [2]. This type of derivative of graphene is named graphene quantum 
dots (GQDs). Many theoretical and experimental studies have been carried out and revealed the excellent properties of GQDs including electronic properties[3-5], magnetic properties[6-8] and photoluminescent properties[9]. Due to these interesting properties, GQDs hold great potential in a variety of advanced applications such as single-electron transistor[3], spintronics [10], energy conversion[5, 11], memory[12], optoelectronics, sensing and bio-imaging [13]. Moreover, compared with their counterparts semiconductor quantum dots, GQDs have competitive advantages with respect to their massless charge carries [14], profound edge effects[15] and low cytotoxicity[16]. These characteristics can provide an environmental friendly and higher performance "dot" in applications where conventional semiconductor quantum dots are currently used.

Issues with the synthesis of GQDs are currently restricting the use of them. The current methods to prepare GQDs could be categorized into two groups, namely top-down and bottom up methods. The top-down methods include hydrothermal cutting $[9,17,18]$, solvothermal cutting $[19,20]$, microwave assisted cleaving[21], electrochemical method[22-24], oxidation of carbon fibre[25] or carbon nanotube[26], exfoliation of graphite nanoparticles[27], cage open of fullerenes[28] and improved Hummer's method[16]. All these methods involve cutting graphitic materials of large sizes into small fragments with sizes in the range of GQDs. In contrast to the relatively extensive research on top-down approaches, just a few researchers have investigated bottom-up synthesis routes despite their superiority in controllable size and uniform structures. The limited studies on bottom-up methods have used organic carbon precursors such as polyphenylene dendritic [11, 29], hexa-perihexabenzocoronene[30], glucose[31]. Although different types of GQDs have been synthesized from these bottom-up methods, the routes of synthesis are quite complex.

Recently Dong et al. [32] have reported a GQDs preparation method by simply heating citric acid at $200^{\circ} \mathrm{C}$. However, this work simply regards the overall products of citric acid thermolysis as GQDs for short reaction durations (30min) and as graphite oxide (GO) for longer duration (2hr). In this work, we provide detailed examination on the relationship between the synthesis conditions 
(temperature, duration) and product properties (physiochemical and optical), which is highly important for obtaining a good understanding of the reaction mechanism and device optimisation strategies. It also intends to provide a detailed account on the structural evolution of TDCAs during thermal decomposition, and an in-depth insight on the structure-property relationship of GQDs, which is a part of TDCAs. In addition to the new findings which could explain the evolution of PL behaviour of TDCAs versus temperature and reaction duration, this study also demonstrates the sensing potential of the as-synthesized GQDs to $\mathrm{Fe}^{3+}$ cation.

\section{Experimental}

2.1 Synthesis of thermally decomposed citric acid (TDCA)

A $50 \mathrm{ml}$ round flask was placed into a silicone oil bath. After the oil bath was heated to desired temperatures (i.e. $180^{\circ} \mathrm{C}, 200^{\circ} \mathrm{C}, 230^{\circ} \mathrm{C}$ and $270^{\circ} \mathrm{C}$ ), $500 \mathrm{mg}$ of citric acid (CA, Chem-Supply) was added into the flask. Upon the completion of reaction for desired duration, $15 \mathrm{ml}$ of sodium hydroxide solution (Chem-Supply) $(0.5 \mathrm{~mol} / \mathrm{L})$ was added to the flask. After the sample was completely dispersed into the solution, the dispersion was transferred to a $100 \mathrm{ml}$ beaker and magnetic stirred for $15 \mathrm{mins}$. The $\mathrm{pH}$ of the final dispersion was then adjusted to 7 .

\subsection{Dialysis of TDCA}

$10 \mathrm{ml}$ of as prepared dispersion was added to a $3.5 \mathrm{kDa}$ dialysis tube membrane (Spectrum Labs). The tube was then immersed into a $20 \mathrm{ml}$ of DI water in a $50 \mathrm{ml}$ beaker for $24 \mathrm{hrs}$. The remaining solution (GQDs solution) in the beaker was collected after the tube was removed. The removed 3.5 $\mathrm{kDa}$ tube membrane along with the remaining solutions inside was then subject to further dialysis for another $24 \mathrm{hrs}$ for purification. The solution collected from the beaker was added into a $1 \mathrm{kDa}$ dialysis tube membrane then allowed for 24 hrs of dialysis to purify the GQDs.

2.3 Preparation of GQDs dispersions with different cations loading 
GQDs dispersions with an absorbance of 0.15 at 370nm under UV/Vis were prepared by diluting the parent solution which was obtained by dialysis of TDCA synthesized at $200^{\circ} \mathrm{C}$ for $30 \mathrm{~min}$. The PH of all the GQDs solutions, for both test of selectivity with different cations and construction of calibration curve for detectable cation $\left(\mathrm{Fe}^{3+}\right)$, were fixed at 6.9 by applying a buffer composed of mixed phosphate salts (Rex, INESA Scientific Instrument). $\mathrm{FeCL}_{3} \cdot 6 \mathrm{H}_{2} \mathrm{O}, \mathrm{CaCl}_{2}, \mathrm{ZnSO}_{4} \cdot 7 \mathrm{H}_{2} \mathrm{O}$, $\mathrm{CuSO}_{4} \cdot 5 \mathrm{H}_{2} \mathrm{O}, \mathrm{MgSO}_{4}$ (Chem-Supply) were added into the GQDs dispersions to form dispersions with the same cations concentration at $0.25 \mathrm{mM}$ for the test of selectivity. Since the amount of added cations in the solutions is very small, the influence to the concentration of GQDs is negligible. For acquisition of the calibration curve of detectable cation $\left(\mathrm{Fe}^{3+}\right)$, a series of loading of $\mathrm{Fe}^{3+}$ from $1 \mu \mathrm{M}$ to $1 \mathrm{mM}$ were adopted.

\subsection{Characterization}

Fluorescence spectra were collected through a Fluorescence spectrometer (Thermo Scientific Lumina). An UV/Vis spectrometer (Agilent 8453) was applied for acquisition of the absorption spectra. Quantum yields (QY) were measured by using quinine sulphite standard solution (quinine sulphite dissolved in $0.05 \mathrm{M} \mathrm{H}_{2} \mathrm{SO}_{4}$, Quantum yield 54\%) as the reference. All samples for quantum yields measurement were prepared by diluting the original samples to the extent that the solution absorbance at $370 \mathrm{~nm}$ is below 0.05 . Elemental compositions were analysed by X-ray photoelectron spectroscopy (XPS, Kratos Axis Ultra). Particle size measurement was conducted via dynamic light scattering (DLS, Malvern ZetasizerNano ZS). Transmission electron microscopy (TEM) of both low (JEM-1010) and high (Philips Tecnai F20) resolutions, along with AFM (NT-MDT NTEGRA Spectra) were employed for the morphology analysis of the as-prepared samples.

\section{Results and Discussion}

\subsection{PL properties of TDCAs}


Excitation independent fluorescence spectra were obtained for TDCAs synthesized at lower temperatures (i.e. $180^{\circ} \mathrm{C}$ and $200^{\circ} \mathrm{C}$ ) and for relatively shorter durations ( $\left.<40 \mathrm{~min}\right)$, however, at lower temperatures for longer durations and at higher temperatures (i.e. $230^{\circ} \mathrm{C}$ and $270^{\circ} \mathrm{C}$ ) even with duration as short as 5min (Fig.S1), samples with excitation-dependent fluorescence spectra were obtained as shown in Figure 1. Figure 2 shows the quantum yields (QYs) for TDCAs synthesized at fixed temperature $200^{\circ} \mathrm{C}$ with varied reaction time (red round dots)) and for fixed duration of $20 \mathrm{~min}$ but at varied temperature (black square). For the fixed temperature at $200^{\circ} \mathrm{C}$, the QYs show a trend of gradual increase till peaking at $30 \mathrm{~min}$ followed by a decrease with the extension of reaction time. On the other hand, for the fixed duration of 20min, QY drops when temperature increases. It is worth noting that for all the excitation-independent samples there is a distinctive peak around $365 \mathrm{~nm}$ in the UV/Vis absorption spectra, however, for all samples with excitation dependence, only featureless decaying absorption curves were observed ( Inserted charts in Fig.1).
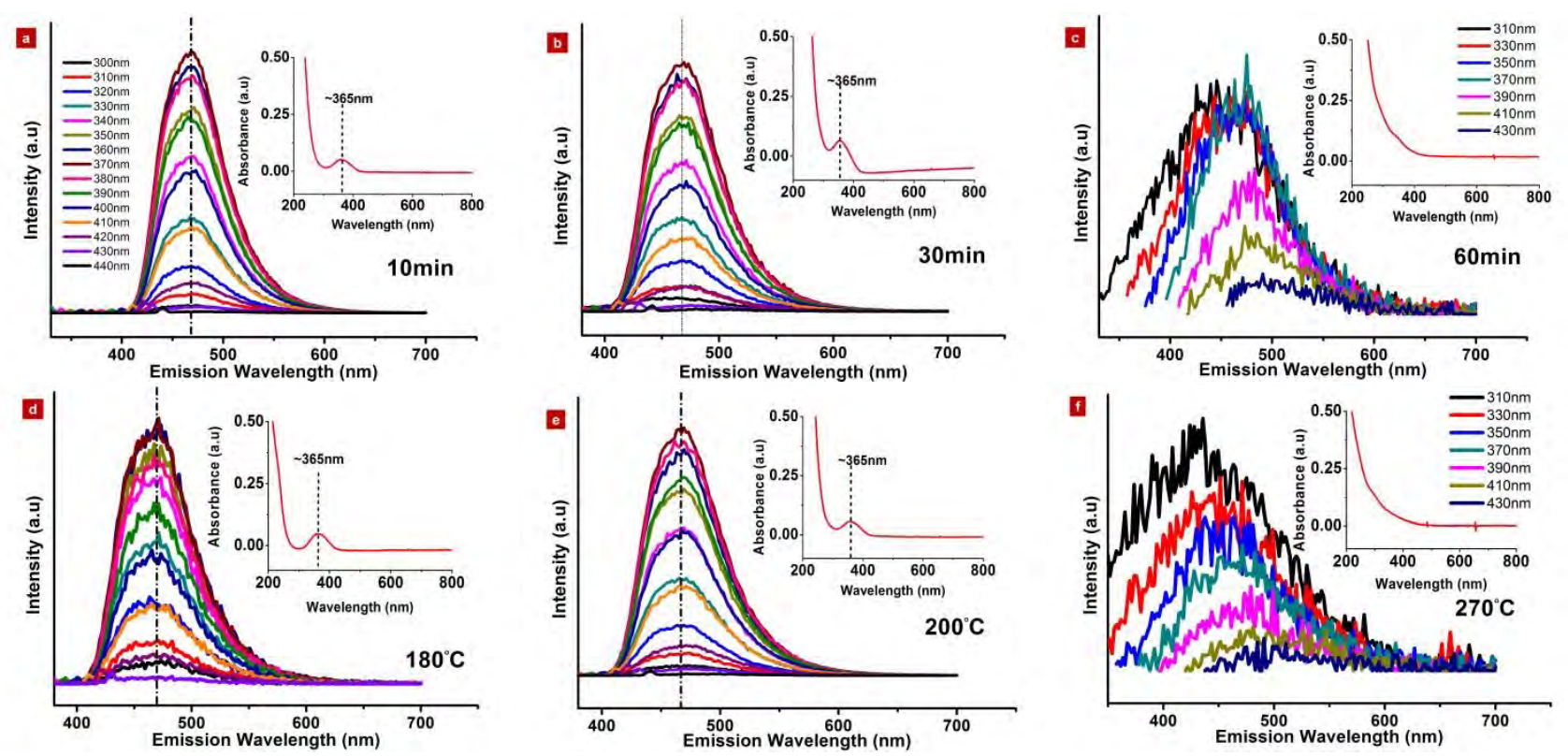

Figure.1 Fluorescence and UV/Vis spectra(inserted) for samples synthesized at $200^{\circ} \mathrm{C}$ for different durations $(\mathrm{a} \sim \mathrm{c})$ and samples synthesized for $20 \mathrm{~min}$ at different temperatures $(\mathrm{d} \sim \mathrm{f})$ 


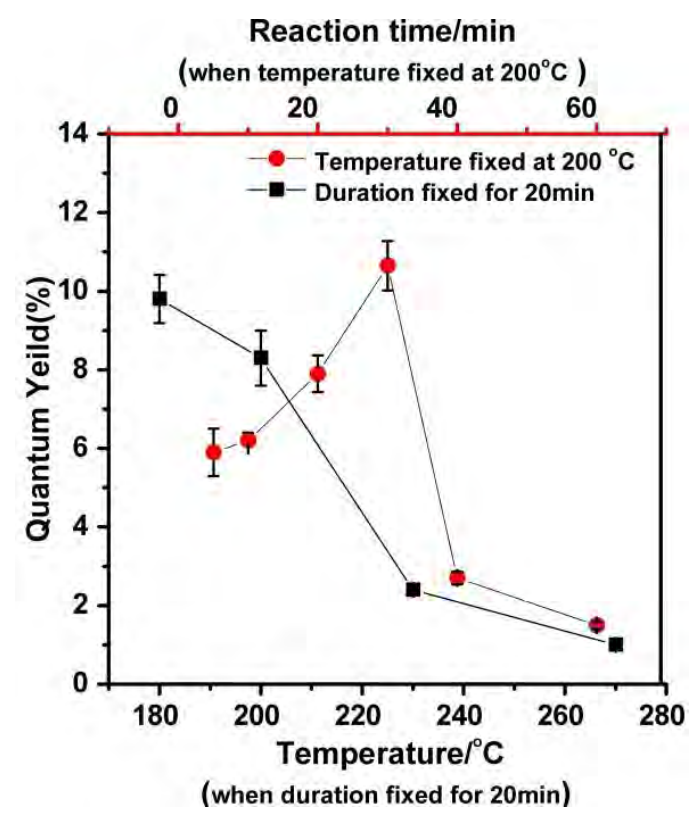

Figure.2 Quantum yields of TDCAs synthesized at fixed temperature $200^{\circ} \mathrm{C}$ and also for fixed duration 20min (Error bar indicating the batch variation)

\subsection{Structural evolution during thermolysis}

Both small individual $\mathrm{sp}^{2}$ domains (GQDs) and large sheet-like structures (see supporting information Fig.S2) were obtained in all the TDCA samples synthesized. When the $200^{\circ} \mathrm{C}-30 \mathrm{~min}$ sample was inspected, small particles with an average height around 1.4nm (Fig.3 a) were detected by AFM. However, HRTEM failed to reveal the shapes and sizes of these small particles, which indicates the size of GQDs synthesized at $200^{\circ} \mathrm{C}$ for $30 \mathrm{~min}$ should be either extremely small or instable under electron beam irradiation. Similarly, all GQDs synthesized at $200^{\circ} \mathrm{C}$ with duration shorter than 30min cannot be observed under HRTEM. However, the large particles as the byproduct bear close resemblance to GO prepared through oxidation of natural graphite with similar shape and wrinkled surface (Fig.S2). In addition, both AFM and TEM images show obvious crosslinking and stacking during the formation of particles (Fig.3 b and c). Hence, it is highly probable that the reaction mechanism of thermal decomposition of citric acid is that citric acid molecules first condense into $\mathrm{sp}^{2}$ domains forming nucleus, followed by subsequent crosslinking and stacking as the reaction proceeds. 
In contrast to TDCA synthesized at $200^{\circ} \mathrm{C}$ for $30 \mathrm{~min}$, the TDCAs synthesized at $270^{\circ} \mathrm{C}$ for $20 \mathrm{mins}$ contain small particles of size 5 7nm with discernable graphitic domains under HRTEM (Fig.2d). The TEM observation for both samples is consistent with our DLS data. In general, the DLS data show multimodal particle size distributions (Fig.3e). In the sample synthesized at $200^{\circ} \mathrm{C}$ for $30 \mathrm{~min}$ (upper diagram in Fig.3e), DLS detected a small portion of narrow distribution with an average size about $0.9 \mathrm{~nm}$ (GQDs) and large particles in the range from $50 \mathrm{~nm}$ to several microns; whilst in the sample synthesized at $270^{\circ} \mathrm{C}$ for $20 \mathrm{~min}$, GQDs with an average size around $7 \mathrm{~nm}$ were detected by DLS (lower diagram in Fig.3e).
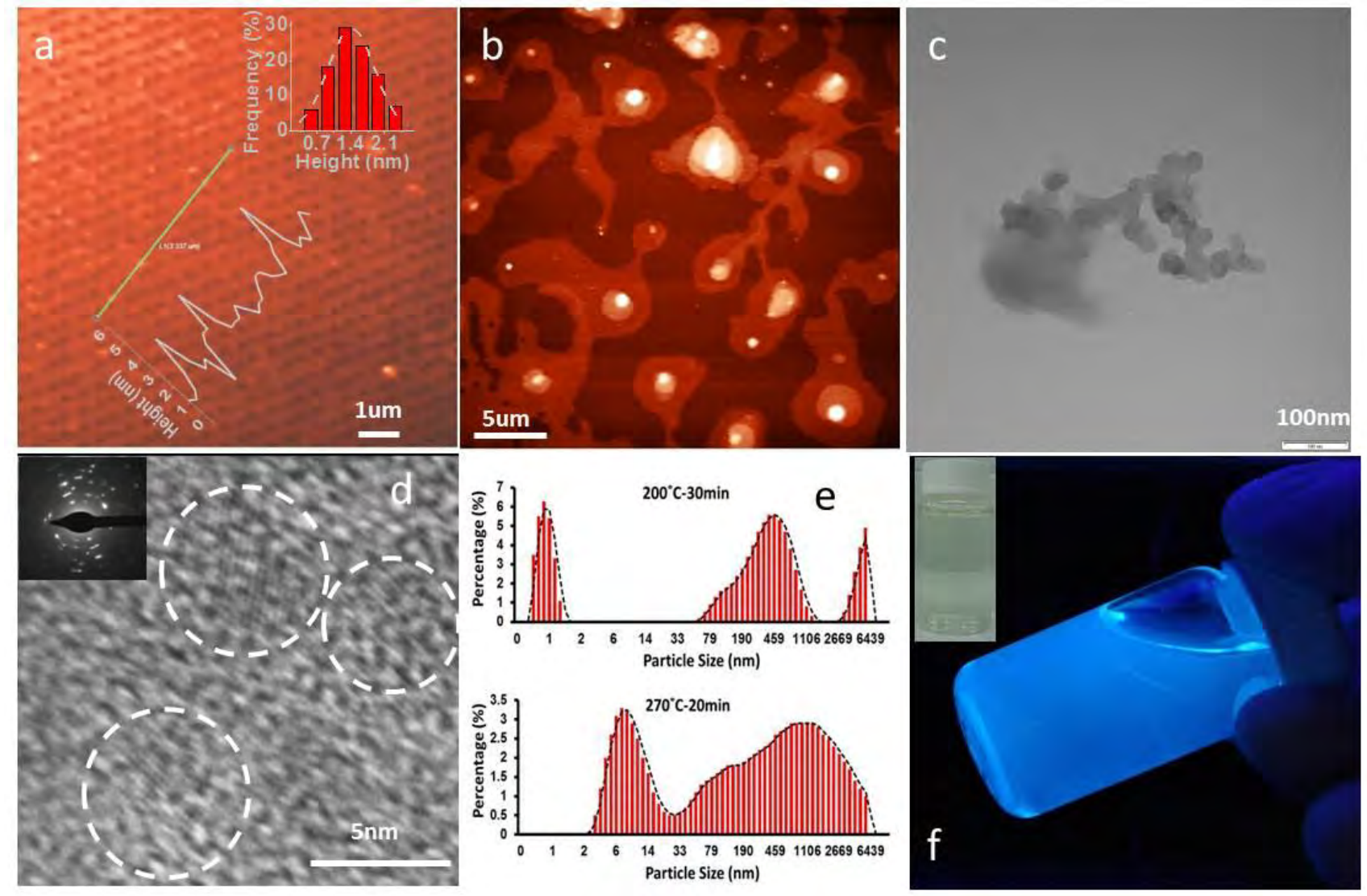

Figure. 3 AFM and TEM images of TDCA sample prepared at $200^{\circ} \mathrm{C}$ for $30 \mathrm{~min}(\mathrm{a} \sim \mathrm{c})$ : a, AFM image of GQDs (insets: upper corner to the right is the height profile distribution which was obtained from over 300 GQDs and lower corner to the left is the height profile of three GQDs), b and c, AFM and TEM image showing crosslinking and stacking of TDCAs ; HRTEM image of GQDs prepared at 
$270^{\circ} \mathrm{C}$ for $20 \mathrm{~min}(\mathrm{~d})$, DLS data for TDCAs prepared at $200^{\circ} \mathrm{C}$ for $30 \mathrm{~min}$ (upper) and $270^{\circ} \mathrm{C}$ for

$20 \mathrm{~min}$ (lower) (e) and; photo of GQDs $\left(200^{\circ} \mathrm{C}\right.$ for $\left.30 \mathrm{~min}\right)$ dispersion in water under $365 \mathrm{~nm} \mathrm{UV}$ lamp (f, inset: the same dispersion under room light).

Secondly, further analysis of the DLS data of all the TDCAs synthesized in our study reveals important information on the population evolution of particles with tuning synthesis conditions. For the samples prepared at the fixed temperature of $200^{\circ} \mathrm{C}$, the percentage of the extremely small GQDs (average size in the range of $0.7 \sim 1 \mathrm{~nm}$ ) contained in the TDCAs increased as reaction proceeded till reaching a peak of $24 \%$ at $30 \mathrm{~min}$, and then dropped accompanied by a rapid percentage rise of much larger-sized particles. Eventually, no GQDs of such small size could be detected after reacting for 60min (Fig.4a). On the other hand, for the fixed reaction duration of 20min, the higher the temperature, the lower the percentage of the tiny GQDs (average size $0.7 \sim 1 \mathrm{~nm}$ ); typically when temperature is higher than $230^{\circ} \mathrm{C}$, no GQDs in size range of $0.7 \sim 1 \mathrm{~nm}$ could be detected (Fig.4b). This size distribution data are intimately related to the PL evolution trend described earlier in 3.1. Namely, high QY, excitation-independent PL can be observed in the TDCAs containing sufficient amount of GQDs sized $0.7 \sim 1 \mathrm{~nm}$. For the TDCAs without the population of $0.7 \sim 1 \mathrm{~nm}$ particle, they exhibit low QY, and excitation-dependent PL. This observation suggests that the evolution trend of the PL properties of TDCAs with temperature and reaction time is likely due to the competition between the small particles with narrow size range (average size $0.7 \sim 1 \mathrm{~nm}$ ) and large particles of broad size distribution. 

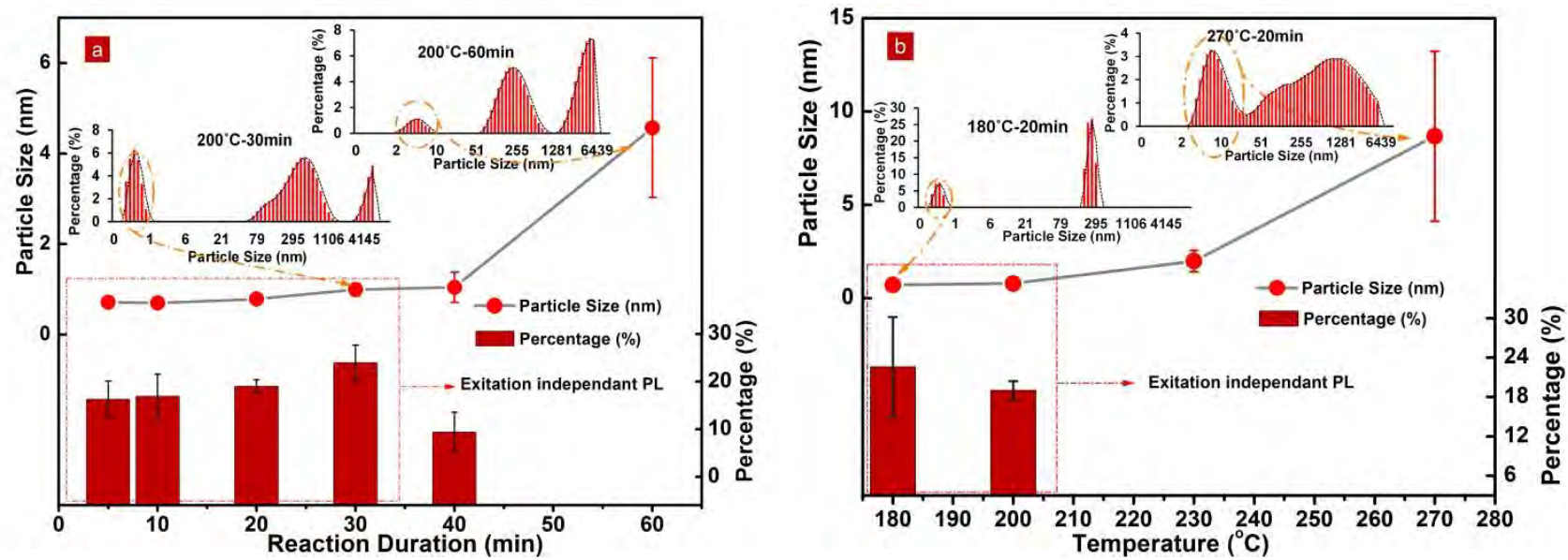

Figure.4 DLS summary on particle size and percentage of small sized GQDs $(0.7 \sim 1 \mathrm{~nm})$ in the TDCAs: a) fixed temperature at $200^{\circ} \mathrm{C}$ and b) fixed reaction duration for $20 \mathrm{~min}$.

According to previous research on the PL of GO [33], both peak wavelength and emission intensity are significantly dominated by the size of the $\mathrm{sp}^{2}$ domains scattered within the basal plane of GO sheets. GQDs are in fact the individual $\mathrm{sp}^{2}$ domains, which also possess size-dependant PL $[25,31]$. GQDs made by the top-down methods have particle size distribution of wide range, therefore their fluorescence spectra exhibit excitation-dependence. However, in our case, the portion of the small particles which is very uniform in terms of size appear to be responsible for the excitationindependent high-QY PL of TDCAs, whereas, the portion of the large particles with inhomogeneous sizes appear to be the source of excitation-dependent low-QY PL of TDCAs. Therefore, it is highly likely that the competition in population between the two parts in the TDCAs determines the PL's excitation energy dependency. To confirm this postulation, dialysis was performed to partition the samples. Through dialysis $(3.5 \mathrm{kDa})$ treatment, it was clearly observed that the fraction which penetrated the dialysis membrane possesses excitation-independent, intense blue emission similar to the original samples, whereas the fraction remained inside the dialysis membrane tube show excitation-dependent features and the luminescence is much weaker in comparison (Fig.5).

Additionally, Figure 5d shows an obvious trend of increased QY with reaction time for the $<3.5 \mathrm{kDa}$ fraction of TDCAs. According to the DLS data, the sizes of the GQDs for the selected reaction 
durations are quite close, therefore this increase of QY of GQDs may be caused by the level of reduction of GQDs as a function of thermolysis duration. Indeed, the carbon-oxygen ratio of GQDs
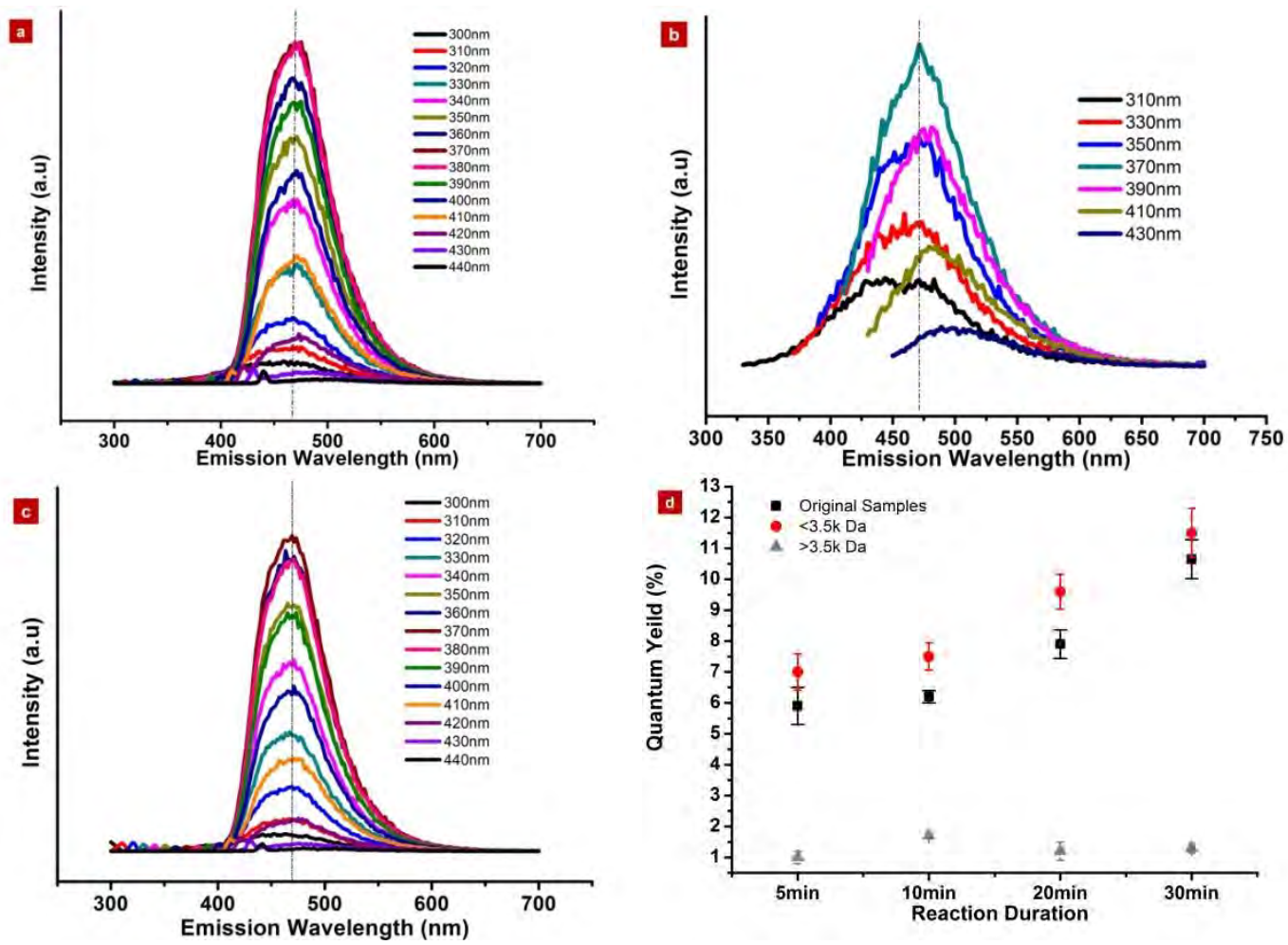

Figure. 5 Fluorescent spectra for TDCA synthesized at $200^{\circ} \mathrm{C}$ for $30 \mathrm{~min}$ with dialysis applied (a c): a) the fraction penetrated dialysis membrane, b) the fraction left inside the dialysis membrane, c) assynthesized TDCA, and; QYs before (Original samples) and after dialysis $(<3.5 \mathrm{k}$ Da and $>3.5 \mathrm{k} \mathrm{Da}$ refers to the fractions penetrated and left inside the dialysis membrane tube respectively) for samples synthesized at $200^{\circ} \mathrm{C}$ for different durations (d)

from XPS analysis, increased with the increase of reaction time (Table 1). This observation shows that as thermolysis proceeds over time, the oxygen content of the GQDs reduces, which resulted in higher QY, consistent with the findings of Zhu et al [20]. The thermally induced reduction in oxygen content can be further specified by analysing the chemical bonds via XPS peak fitting. As shown in Figure 6, at a fixed temperature of $200^{\circ} \mathrm{C}$, with the extension of reaction duration, the amount of both hydroxyl (-C-OH) and carboxyl groups $(\mathrm{O}=\mathrm{C}-\mathrm{OH})$ decreased, whereas the amount of carbon-carbon 
bonding $(\mathrm{C} / \mathrm{C}=\mathrm{C})$ increased. Detailed percentage of all observed chemical bonds in the analysed samples were provided in Table 1. It should also be noted that there is a gradual increase of carbonyl group content, which can be ascribed to the conversion of carboxyl group possessed by the precursor citric acid.
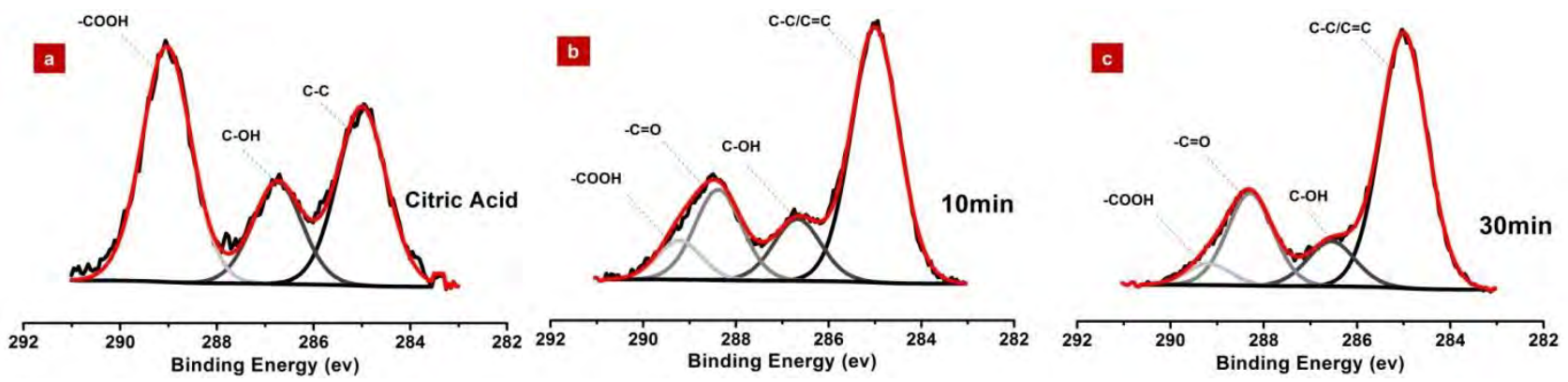

Figure.6 XPS peaks fitting for pure citric acid(a), GQDs $(<3.5 \mathrm{~K} \mathrm{Da})$ prepared at $200^{\circ} \mathrm{C}$ for different durations

\begin{tabular}{ccccccc}
\hline Durations & $\mathrm{C}: \mathrm{O}$ & $\mathrm{C}-\mathrm{C} / \mathrm{C}=\mathrm{C}$ & $\mathrm{C}-\mathrm{O}$ & $\mathrm{O}=\mathrm{C}$ & $\mathrm{COOH}$ & $\mathrm{QY}$ \\
& & $285 \mathrm{ev}$ & $\sim 286.4 \mathrm{ev}$ & $\sim 288.3 \mathrm{ev}$ & $\sim 289.1 \mathrm{ev}$ & \\
\hline 0min & 1.1 & 34.6 & 19.7 & & 45.6 & \\
$5 \mathrm{~min}$ & 1.5 & 50.4 & 15.8 & 19.9 & 13.9 & $7.0 \pm 0.59$ \\
$10 \mathrm{~min}$ & 2.3 & 57.5 & 14.0 & 20.4 & 8.1 & $7.5 \pm 0.44$ \\
$20 \mathrm{~min}$ & 2.4 & 61.9 & 11.6 & 21.3 & 5.2 & $9.6 \pm 0.57$ \\
$30 \mathrm{~min}$ & 2.3 & 62.1 & 11.1 & 21.9 & 4.9 & $11.5 \pm 0.80$
\end{tabular}

Table.1 C1s bonding states assignment from XPS peak fitting, carbon-oxygen ratio and PL quantum yields for GQDs synthesized at $200^{\circ} \mathrm{C}$ for different durations

For a fixed duration at 20min, GQDs with size $<3.5 \mathrm{kDa}$ could only be obtained at low temperatures $\left(180^{\circ} \mathrm{C}, 200^{\circ} \mathrm{C}\right)$, therefore, the XPS data of as prepared TDCA samples were measured for analysis of chemistry evolution with varying temperatures. As displayed in Figure 7, decrease in hydroxyl and carboxyl groups, and increase of the carbon-carbon bonding $(\mathrm{C} / \mathrm{C}=\mathrm{C})$ is observed (see Table 2 for specific contents of different bonding states), similar to the fixed temperature case. However, the 
amount of carbonyl groups experienced a decline, which means that higher temperature could remove the carbonyl groups better than the low temperature and ensures better crystallization. As for the QYs of those samples (Table 2), although the degree of reduction is also raised with elevated temperature, it hardly improved the QYs of TDCAs because the effect is rapidly overtaken by the percentage increase of large particles. Therefore, a rapid diminishing trend of QY with temperature rise was observed.
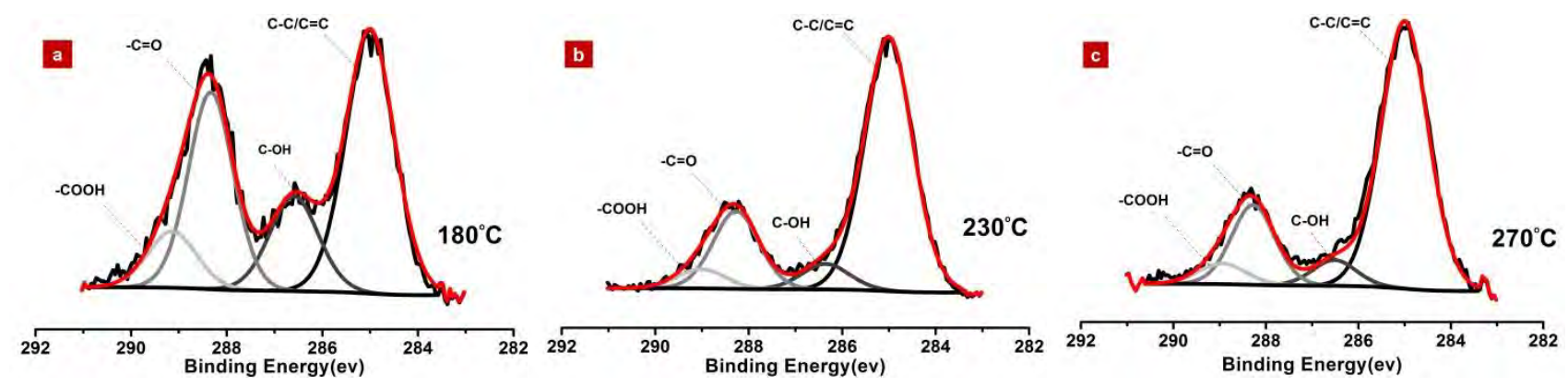

Figure.7 XPS peaks fitting for TDCAs prepared at different temperatures for fixed duration at 20min

\begin{tabular}{ccccccc}
\hline Temperatures & $\mathrm{C}: \mathrm{O}$ & $\begin{array}{c}\mathrm{C}-\mathrm{C} / \mathrm{C}=\mathrm{C} \\
\sim 285 \mathrm{ev}\end{array}$ & $\begin{array}{c}\mathrm{C}-\mathrm{O} \\
\sim 286.4 \mathrm{ev}\end{array}$ & $\begin{array}{c}\mathrm{O}=\mathrm{C} \\
\sim 288.3 \mathrm{ev}\end{array}$ & $\begin{array}{c}\mathrm{COOH} \\
\sim 289.1 \mathrm{ev}\end{array}$ & $\mathrm{QY}$ \\
\hline $180^{\circ} \mathrm{C}$ & 1.8 & 44.1 & 15.9 & 30.5 & 9.5 & $10.4 \pm 0.61$ \\
$230^{\circ} \mathrm{C}$ & 2.5 & 68.0 & 6.9 & 20.0 & 5.2 & $2.4 \pm 0.70$ \\
$270^{\circ} \mathrm{C}$ & 2.9 & 69.5 & 6.2 & 19.0 & 5.4 & $1.0 \pm 0.07$ \\
\hline
\end{tabular}

Table.2 C1s bonding states assignment from XPS peak fitting, carbon-oxygen ratio and PL quantum yields for TDCAs synthesized for 20min at different temperatures

From the evolution trend of the PL properties as a function of the structure of TDCAs, a clear understanding could be obtained on the thermal decomposition process, as illustrated in Figure 8 . Firstly, higher temperature results in fiercer reactions. It takes less time for GQDs with average size in the range of $0.7 \sim 1 \mathrm{~nm}$ to nucleate from citric acid molecules and then crosslink and stack to form larger particles. At higher temperature, most of the tiny GQDs would be consumed very quickly to form particles with larger inhomogeneous sizes than in the case of lower temperatures. Therefore, the 
excitation-dependent, low QY emission from the larger-sized particles dominates the PL of TDCAs. On the other hand, for a fixed temperature, with the extension of reaction time, more citric acid molecules will be consumed to form GQDs, meanwhile GQDs will crosslink and stack to form larger particles. The longer the duration, more large particles will be constructed, eventually with few tiny GQDs, which possess excitation-independent intense PL, left inside the TDCAs. Hence the excitation-dependent low QY emission from the larger-inhomogenesously-sized particles becomes dominant. Only for those cases at relatively lower temperatures $\left(180^{\circ} \mathrm{C}, 200^{\circ} \mathrm{C}\right)$ and for relatively shorter durations, there are sufficient quantities of highly luminescent, uniformly-sized GQDs within the TDCAs which therefore exhibit strong excitation-independent PL.

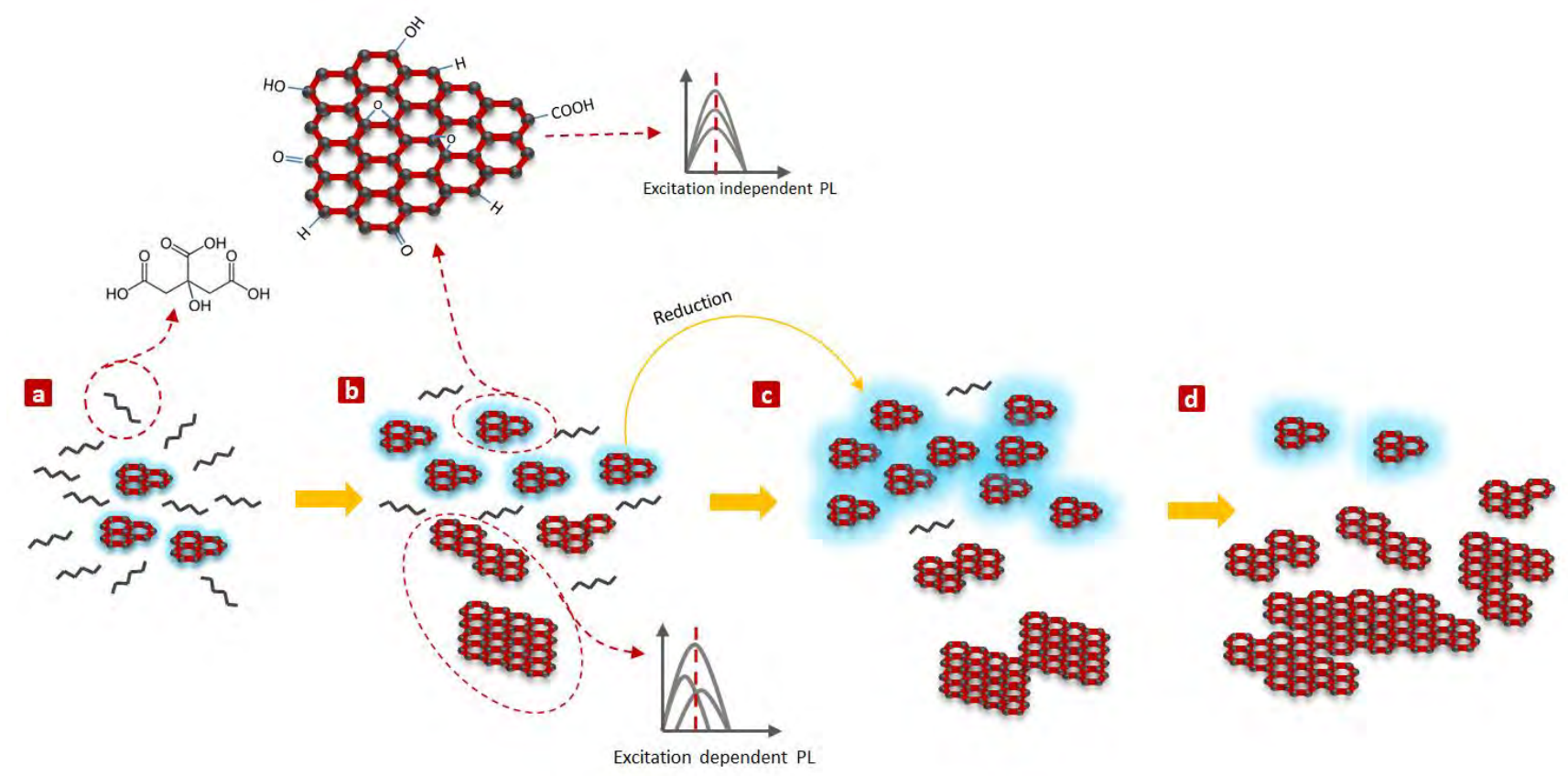

Figure.8 Schematic representation of the thermal decomposition process of CA: a, GQDs nucleate from CA molecules; $b$, further GQDs nucleation with more CA molecules consumed, meanwhile GQDs crosslinking and stacking to form large particles with inhomogeneous sizes which possess excitation dependant PL; c, when the amount of GQDs reaches the maximum value, PL of the thermal decomposed product reached optimal level with highest QY; d, with extension of reaction duration, most of the GQDs are consumed for formation of large particles, therefore, excitation- 
dependent PL of those large particles with ununiformed sizes dominates the PL of TDCAs. From a d, GQDs are also reduced to a lower oxygen content levels, which in principle improves the PL of the thermal decomposition process.

\subsection{Cation sensing}

As shown in the XPS study, the GQDs possess abundant negative charged surface moieties, such as hydroxyl and carboxyl groups. The interaction between these functional groups and cations may influence the PL of GQDs, which can be used for sensor development. Figure 9a shows the PL intensity of GQDs solutions synthesized at $200^{\circ} \mathrm{C}$ for $30 \mathrm{~min}$ with different cations of the same loading of $0.25 \mathrm{mM} / \mathrm{L}$. It is apparently that all tested cations possess certain degree of quenching effect on PL of GQDs, however, $\mathrm{Fe}^{3+}$ has the most significant quenching efficiency than the rest, which means that raw GQDs have excellent selectivity towards $\mathrm{Fe}^{3+}$. Figure $9 \mathrm{~b}$ presents the correlation between $\mathrm{Fe}^{3+}$ concentration and $\mathrm{PL}$ of GQDs dispersions. A fine linear relationship between $\mathrm{Fe}^{3+}$ concentration and PL intensity of GQDs dispersions could be constructed.
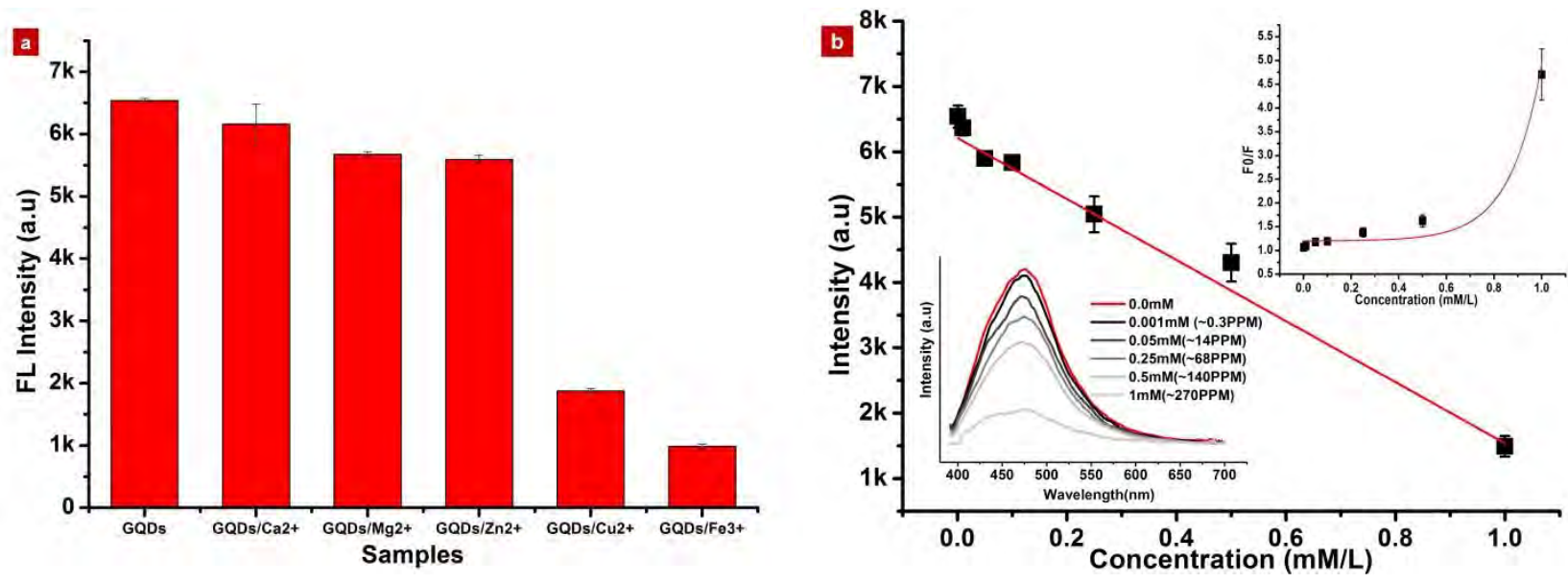

Figure.9 $\mathrm{Fe}^{3+}$ sensing illustration: a, Quenching capability of different cations on PL of GQDs; $b$, linear relationship between $\mathrm{Fe}^{3+}$ concentration and PL intensity of GQDs. Inserted upper corner to the right is the Stern-Volmer plot and lower corner to the left is the fluorescence spectra of GQDs solutions with different $\mathrm{Fe}^{3+}$ concentration 
To understand the quenching mechanism of the $\mathrm{Fe}^{3+}$ ions quenching of GQDs PL, the Stern-volmer plot was plotted (Inserted figure in Fig. $8 \mathrm{~b}$ at the upper corner to the right), showing a curve shape. This indicates that a combined quenching mechanism (both static and collisional) [34] is responsible for the PL quenching. As shown in Figure 10b, static quenching effect of $\mathrm{Fe}^{3+}$ on the PL of GQDs involves the formation of non-radiative complex ground states between GQDs and $\mathrm{Fe}^{3+}$ through ionic bonding, which results in non-radiative decay upon absorption of incident photons. Therefore, the PL intensity of GQDs is quenched. Whereas, in collisional quenching, upon absorption of photons, electrons are promoted from ground states to exited states in GQDs; however, when electrons of GQDs return to the ground state of GQDs, instead of releasing energy through photons, the energy is absorbed by $\mathrm{Fe}^{3+}$, as shown in Figure 10c. Observable PL quenching could be obtained down to a $\mathrm{Fe}^{3+}$ concentration of $0.3 \mathrm{ppm}$ and up to $270 \mathrm{ppm}$ (Inserted figure in Fig.9b at the lower corner to the left), which illustrates a high sensitivity and a wide detective range, although the theoretical determination limit derived from the calibration curve is around 6.47ppm (see Supplementary Information for determination of detection limit). Thus, raw GQDs could be used as a sensing material for $\mathrm{Fe}^{3+}$ with excellent selectivity and sensitivity. It can be envisioned that through appropriate surface functionalization, raw GQDs synthesized in this investigation could be further engineered to tailor for various sensing purposes including not only cations but also organic chemicals, which is currently under investigation. 


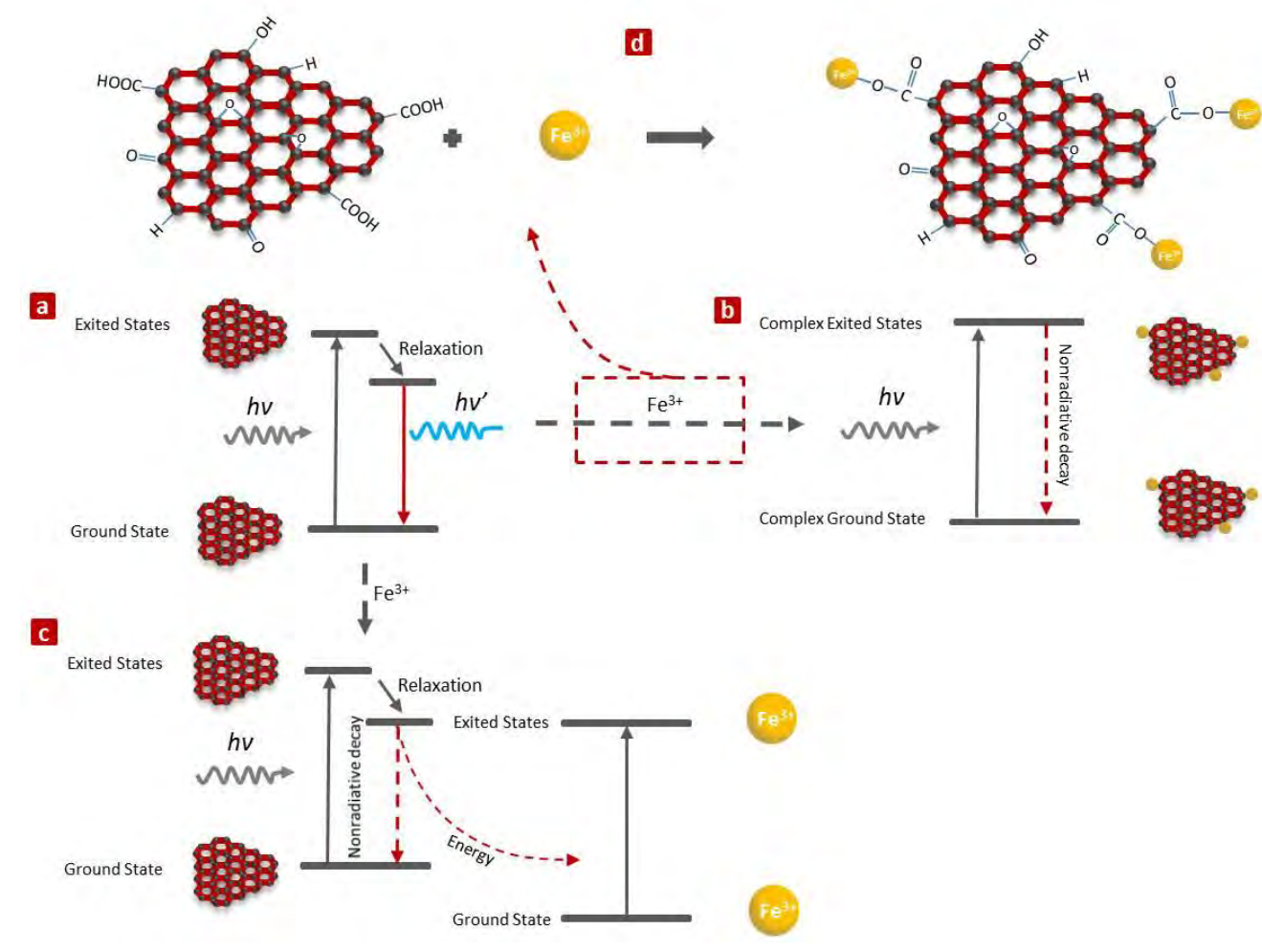

Figure.10 Schematic representation of $\mathrm{Fe}^{3+}$ quenching mechanisms on PL of GQDs: a, natural emission of raw GQDs upon absorption of incident photons; $b$, static quenching effect of $\mathrm{Fe}^{3+}$ on PL of GQDs; c, collisional quenching effects of $\mathrm{Fe}^{3+}$ on PL of GQDs; $\mathrm{d}$, the possible formation mechanism of complex between GQDs and $\mathrm{Fe}^{3+}$ via ionic bonding as a result of negative charging of raw GQDs.

\section{Conclusion}

By tuning the synthesis conditions, the TDCAs exhibit either excitation-independent or excitationdependent fluorescence spectra and varied QYs. These features are majorly determined by the quantitative competition between the GQDs and the large-sized GO particles in the thermally decomposed products. The fraction of small uniformly sized GQDs (average size $0.7 \mathrm{~nm} \sim 1 \mathrm{~nm}$ ) is responsible for the excitation-independent, high QY, blue fluorescence of TDCAs; whereas the fraction of large inhomogeneously sized sheet structures dominate excitation-dependent, low QY fluorescence emission of TDCAs. When a sufficient quantity of small GQDs is obtained in the 
TDCAs, they exhibit high QY, excitation-independent photoluminescence, otherwise they show low QY, excitation-dependent photoluminescence. In addition to elucidating the structural evolution of GQDs during thermal decomposition of CA and its relationship to the PL properties, we have also demonstrated that the raw GQDs synthesized in this study can serve as an excellent fluorescence sensor for trivalent iron cation with good selectivity and a high sensitivity down to $0.3 \mathrm{ppm}$.

\section{Acknowledgement}

This study was supported by a Griffith University International Postgraduate Scholarship and a CSIRO OCE top-up scholarship. The authors would like to thank the technical support from the Centre for Microscopy and Microanalysis at the University of Queensland.

\section{Supporting Information}

Detailed information on excitation-dependent photoluminescence, GO morphology and detection limit determination are provided. 


\section{References}

[1] Ponomarenko LA, Schedin F, Katsnelson MI, Yang R, Hill EW, Novoselov KS, et al. Chaotic Dirac billiard in graphene quantum dots. Science. 2008;320(5874):356-8.

[2] Novoselov KS, Geim AK, Morozov SV, Jiang D, Zhang Y, Dubonos SV, et al. Electric field effect in atomically thin carbon films. Science. 2004;306(5696):666-9.

[3] Barreiro A, van der Zant HS, Vandersypen LM. Quantum dots at room temperature carved out from few-layer graphene. Nano letters. 2012;12(12):6096-100.

[4] Mandal B, Sarkar S, Sarkar P. Exploring the electronic structure of graphene quantum dots. Journal of Nanoparticle Research. 2012;14(12).

[5] Yan X, Li B, Cui X, Wei Q, Tajima K, Li L-s. Independent Tuning of the Band Gap and Redox Potential of Graphene Quantum Dots. The Journal of Physical Chemistry Letters. 2011;2(10):1119-24.

[6] Agapito LA, Kioussis N, Kaxiras E. Electric-field control of magnetism in graphene quantum dots: $A b$ initio calculations. Physical Review B. 2010;82(20).

[7] Espinosa-Ortega T, Luk'yanchuk IA, Rubo YG. Magnetic properties of graphene quantum dots. Physical Review B. 2013;87(20).

[8] Ma W-L, Li S-S. Electric-field-induced spin depolarization in graphene quantum dots. Physical Review B. 2012;86(4).

[9] Pan D, Zhang J, Li Z, Wu M. Hydrothermal route for cutting graphene sheets into blue-luminescent graphene quantum dots. Advanced materials. 2010;22(6):734-8.

[10] Ezawa M. Quasi-ferromagnet spintronics in the graphene nanodisc-lead system. New Journal of Physics. 2009;11(9):095005.

[11] Yan X, Cui X, Li B, Li LS. Large, solution-processable graphene quantum dots as light absorbers for photovoltaics. Nano letters. 2010;10(5):1869-73.

[12] Kou L, Li F, Chen W, Guo T. Synthesis of blue light-emitting graphene quantum dots and their application in flexible nonvolatile memory. Organic Electronics. 2013;14(6):1447-51.

[13] Shen J, Zhu Y, Yang X, Li C. Graphene quantum dots: emergent nanolights for bioimaging, sensors, catalysis and photovoltaic devices. Chemical communications. 2012;48(31):3686-99.

[14] Rozhkov AV, Giavaras G, Bliokh YP, Freilikher V, Nori F. Electronic properties of mesoscopic graphene structures: Charge confinement and control of spin and charge transport. Physics Reports. 2011;503(2-3):77114.

[15] Girit CO, Meyer JC, Erni R, Rossell MD, Kisielowski C, Yang L, et al. Graphene at the edge: stability and dynamics. Science. 2009;323(5922):1705-8.

[16] Sun Y, Wang S, Li C, Luo P, Tao L, Wei Y, et al. Large scale preparation of graphene quantum dots from graphite with tunable fluorescence properties. Physical chemistry chemical physics : PCCP. 2013;15(24):9907-13.

[17] Pan D, Guo L, Zhang J, Xi C, Xue Q, Huang H, et al. Cutting sp2 clusters in graphene sheets into colloidal graphene quantum dots with strong green fluorescence. Journal of Materials Chemistry. 2012;22(8):3314.

[18] Shen J, Zhu Y, Yang X, Zong J, Zhang J, Li C. One-pot hydrothermal synthesis of graphene quantum dots surface-passivated by polyethylene glycol and their photoelectric conversion under near-infrared light. New Journal of Chemistry. 2012;36(1):97.

[19] Zhu S, Zhang J, Qiao C, Tang S, Li Y, Yuan W, et al. Strongly green-photoluminescent graphene quantum dots for bioimaging applications. Chemical communications. 2011;47(24):6858-60.

[20] Zhu S, Zhang J, Liu X, Li B, Wang X, Tang S, et al. Graphene quantum dots with controllable surface oxidation, tunable fluorescence and up-conversion emission. RSC Advances. 2012;2(7):2717.

[21] Li L-L, Ji J, Fei R, Wang C-Z, Lu Q, Zhang J-R, et al. A Facile Microwave Avenue to Electrochemiluminescent Two-Color Graphene Quantum Dots. Advanced Functional Materials. 2012;22(14):2971-9.

[22] Li Y, Hu Y, Zhao Y, Shi G, Deng L, Hou Y, et al. An electrochemical avenue to green-luminescent graphene quantum dots as potential electron-acceptors for photovoltaics. Advanced materials. 2011;23(6):776-80. 
[23] Shinde DB, Pillai VK. Electrochemical preparation of luminescent graphene quantum dots from multiwalled carbon nanotubes. Chemistry. 2012;18(39):12522-8.

[24] Zhang M, Bai L, Shang W, Xie W, Ma H, Fu Y, et al. Facile synthesis of water-soluble, highly fluorescent graphene quantum dots as a robust biological label for stem cells. Journal of Materials Chemistry. 2012;22(15):7461.

[25] Peng J, Gao W, Gupta BK, Liu Z, Romero-Aburto R, Ge L, et al. Graphene quantum dots derived from carbon fibers. Nano letters. 2012;12(2):844-9.

[26] Minati L, Torrengo S, Maniglio D, Migliaresi C, Speranza G. Luminescent graphene quantum dots from oxidized multi-walled carbon nanotubes. Materials Chemistry and Physics. 2012;137(1):12-6.

[27] Liu F, Jang MH, Ha HD, Kim JH, Cho YH, Seo TS. Facile synthetic method for pristine graphene quantum dots and graphene oxide quantum dots: origin of blue and green luminescence. Advanced materials. 2013;25(27):3657-62.

[28] Lu J, Yeo PSE, Gan CK, Wu P, Loh KP. Transforming C-60 molecules into graphene quantum dots. Nat Nanotechnol. 2011;6(4):247-52.

[29] Yan X, Cui X, Li LS. Synthesis of Large, Stable Colloidal Graphene Quantum Dots with Tunable Size. J Am Chem Soc. 2010;132(17):5944-+.

[30] Liu R, Wu D, Feng X, Mullen K. Bottom-up fabrication of photoluminescent graphene quantum dots with uniform morphology. Journal of the American Chemical Society. 2011;133(39):15221-3.

[31] Tang L, Ji R, Li X, Teng KS, Lau SP. Size-Dependent Structural and Optical Characteristics of GlucoseDerived Graphene Quantum Dots. Particle \& Particle Systems Characterization. 2013;30(6):523-31.

[32] Dong $Y$, Shao J, Chen $\mathrm{C}$, Li H, Wang R, Chi Y, et al. Blue luminescent graphene quantum dots and graphene oxide prepared by tuning the carbonization degree of citric acid. Carbon. 2012;50(12):4738-43.

[33] Eda G, Lin YY, Mattevi C, Yamaguchi H, Chen HA, Chen IS, et al. Blue photoluminescence from chemically derived graphene oxide. Adv Mater. 2010;22(4):505-9.

[34] Lakowicz JR. Principles of fluorescence spectroscopy. New York: Plenum Press; 1983. 


\title{
Structural evolution of graphene quantum dots during thermal decomposition of citric acid and the corresponding photoluminescence
}

\author{
Shujun Wang ${ }^{1}$, Ivan Cole ${ }^{2}$ and Qin $\mathrm{Li}^{1 *}$
}

1. Environmental Engineering \& Queensland Miro- and Nanotechnology Centre, Griffith University, Nathan Campus, Brisbane, QLD 4111, Australia

2. CSIRO Materials Science and Engineering - Clayton, Gate 5, Normanby Road Clayton VIC 3168, Australia

\begin{abstract}
The thermally decomposed citric acid (TDCA) possesses either excitation-dependent or excitationindependent fluorescence as well as different quantum yields with varying synthesis conditions (i.e. temperature and reaction duration). These photoluminescent (PL) properties were found to be mainly determined by the quantitative competition between the graphene quantum dots (average size in the range $0.7 \sim 1 \mathrm{~nm}$ ) and the large-inhomogeneously-sized particles. Thermal induced reduction of oxygen containing functionalities leads to an enhancing effect to the PL of GQDs. The study reveals the structural evolution of the GQDs upon thermal treatment and attempts to establish its relationship to the PL property. The GQDs synthesized in this study are excellent sensing materials for trivalent iron cation with both notable selectivity and sensitivity.
\end{abstract}

\section{Introduction}

Graphene sheets smaller than 100nm show quantum confinement behaviour which generates band gaps in their electronic band structures[1] in contrast to the well-known zero-gap feature of graphene sheets with much larger sizes [2]. This type of derivative of graphene is named graphene quantum dots (GQDs). Many theoretical and experimental studies have been carried out and revealed the excellent properties of GQDs including tuneable electronic properties[3-5], tuneable magnetic 
properties[6-8] and photoluminescent properties[9]. Due to these interesting properties, GQDs hold great potential in a variety of advanced applications such as single-electron transistor[3], spintronics [10], energy conversion[5, 11], memory[12], optoelectronics, sensing and bioimaging [13]. Moreover, compared with their counterparts semiconductor quantum dots, GQDs have competitive advantages with respect to their massless charge carries [14], profound edge effects[15] and low cytotoxicity[16]. These characteristics can provide an environmental friendly and higher performance "dot" in applications where conventional semiconductor quantum dots are currently used.

Issues with the synthesis of GQDs are currently restricting the use of them. The current methods to prepare GQDs could be categorized into two groups, namely top-down and bottom up methods. The top-down methods include hydrothermal cutting $[9,17,18]$, solvothermal cutting $[19,20]$,microwave assisted cleaving[21], electrochemical method[22-24], oxidation of carbon fibre[25] or carbon nanotube[26], exfoliation of graphite nanoparticles[27], cage open of fullerenes[28] and improved Hummer's method[16]. All these methods involve cutting graphitic materials of large sizes into small fragments with sizes in the range of GQDs. In contrast to the relatively extensive research on top-down approaches, just a few researchers have investigated bottom-up synthesis routes despite their superiority in controllable size and uniform structures. The limited studies on bottom-up methods have used organic carbon precursors such as polyphenylene dendritic [11, 29], hexa-perihexabenzocoronene[30], glucose[31]. Although different types of GQDs have been synthesized from these bottom-up methods, the routes of synthesis are quite complex.

Recently Dong et al. [32] have reported a GQDs preparation method by simply heating citric acid at $200^{\circ} \mathrm{C}$. However, this work simply regards the overall products of citric acid thermolysis as GQDs for short reaction durations (30min) and as graphite oxide (GO) for longer duration (2hr). In this work, we provide detailed examination on the relationship between synthesis conditions (temperature, duration) and product properties (physiochemical and optical), which is highly important for 
obtaining a good understanding of the reaction mechanism and device optimisation strategies. It also intends to provide a detailed account on the structural evolution of TDCAs during thermal decomposition, and an in-depth insight on the structure-property relationship of GQDs, which is a part of TDCAs. In addition to the new findings which could explain the evolution of PL behaviour of TDCAs versus temperature and reaction duration, this study also demonstrates the sensing potential of the as-synthesized GQDs to $\mathrm{Fe}^{3+}$ cation.

\section{Experimental}

2.1 Synthesis of thermally decomposed citric acid (TDCA)

A $50 \mathrm{ml}$ round flask was placed into a silicone oil bath. After the oil bath was heated to desired temperatures (i.e. $180^{\circ} \mathrm{C}, 200^{\circ} \mathrm{C}, 230^{\circ} \mathrm{C}$ and $270^{\circ} \mathrm{C}$ ), $500 \mathrm{mg}$ of citric acid (CA, Chem-Supply) was added into the flask. Upon the completion of reaction for desired duration, $15 \mathrm{ml}$ of sodium hydroxide solution (Chem-Supply) $(0.5 \mathrm{~mol} / \mathrm{L})$ was added to the flask. After the sample was completely dispersed into the solution, the dispersion was transferred to a $100 \mathrm{ml}$ beaker and magnetic stirred for $15 \mathrm{mins}$. The $\mathrm{pH}$ of the final dispersion was then adjusted to 7 .

\subsection{Dialysis of TDCA}

$10 \mathrm{ml}$ of as prepared dispersion was added to a $3.5 \mathrm{kDa}$ dialysis tube membrane (Spectrum Labs).The tube was then immersed into a $20 \mathrm{ml}$ of DI water in a $50 \mathrm{ml}$ beaker for $24 \mathrm{hrs}$. The remaining solution (GQDs solution) in the beaker was collected after the tube was removed. The removed 3.5 $\mathrm{kDa}$ tube membrane along with the remaining solutions inside was then subject to further dialysis for

another $24 \mathrm{hrs}$ for purification. The solution collected from the beaker was added into a $1 \mathrm{kDa}$ dialysis tube membrane then allowed for $24 \mathrm{hrs}$ of dialysis to purify the GQDs.

2.3 Preparation of GQDs dispersions with different cations loading 
GQDs dispersions with an absorbance of $0.15 \%$ at $370 \mathrm{~nm}$ under UV/Vis were prepared by diluting the parent solution which was obtained by dialysis of TDCA synthesized at $200^{\circ} \mathrm{C}$ for $30 \mathrm{~min}$. $\mathrm{FeCL}_{3} \cdot 6 \mathrm{H}_{2} \mathrm{O}, \mathrm{CaCl} 2, \mathrm{ZnSO}_{4} \cdot 7 \mathrm{H}_{2} \mathrm{O}, \mathrm{CuSO}_{4} \cdot 5 \mathrm{H}_{2} \mathrm{O}, \mathrm{MgSO}_{4}$ (Chem-Supply) were added into the GQDs dispersions to form dispersions with the same cations concentration at $0.25 \mathrm{mM}$ for the selectivity tests. For detectable cations a series of loading from $1 \mu \mathrm{M}$ to $1 \mathrm{mM}$ were prepared to characterise the sensitivity of GQDs to them.

\subsection{Characterization}

Fluorescent spectra were collected through a Fluorescence spectrometer (Thermo Scientific Lumina). An UV/Vis spectrometer (Agilent 8453) was applied for acquisition of the absorption spectra. Quantum yields (QY) were measured by using quinine sulphite (Quantum yield 54\%) as the reference. All samples for quantum yield measurement were prepared by diluting the original samples to the extent that the solution absorbance at $370 \mathrm{~nm}$ is below $0.05 \%$. Elemental compositions were analysed by X-ray photoelectron spectroscopy (XPS, Kratos Axis Ultra).Particle size measurement was conducted via dynamic light scattering (DLS, Malvern ZetasizerNano ZS). Transmission electron microscopy (TEM) both low (JEM-1010) and high (Philips Tecnai F20) resolution, along with AFM (NT-MDT NTEGRA Spectra) were adopted for the morphology analysis of the as-prepared samples.

\section{Results and Discussion}

\subsection{PL properties of TDCAs}

Excitation independent fluorescence spectra were obtained for TDCAs synthesized at lower temperatures (i.e. $180^{\circ} \mathrm{C}$ and $200^{\circ} \mathrm{C}$ ) and for relatively shorter durations $(<40 \mathrm{~min})$, however, at lower temperatures for longer durations and at higher temperatures (i.e $230^{\circ} \mathrm{C}$ and $270^{\circ} \mathrm{C}$ ) even with duration as short as 5min (Fig.S1), samples with excitation-dependant fluorescence spectra were 
obtained as shown in Figure 1. Figure 2 shows the quantum yields (QY) for TDCAs synthesized at fixed temperature $200^{\circ} \mathrm{C}$ with varied reaction time (top row) and for fixed duration of $20 \mathrm{~min}$ but at varied temperature (bottom row). For the fixed temperature at $200^{\circ} \mathrm{C}$, the QYs show a trend of gradual increase till peaking at $30 \mathrm{~min}$ followed by a decrease with the extension of reaction time. On the other hand, for the fixed duration 20min, QY drops when temperature increases. It is worth noting that for all the excitation-independent samples there is a distinctive peak around $365 \mathrm{~nm}$ in the UV/Vis absorption spectra, however, for all samples with excitation dependence, only featureless decaying absorption curves were observed ( Inserted charts in Fig.1).
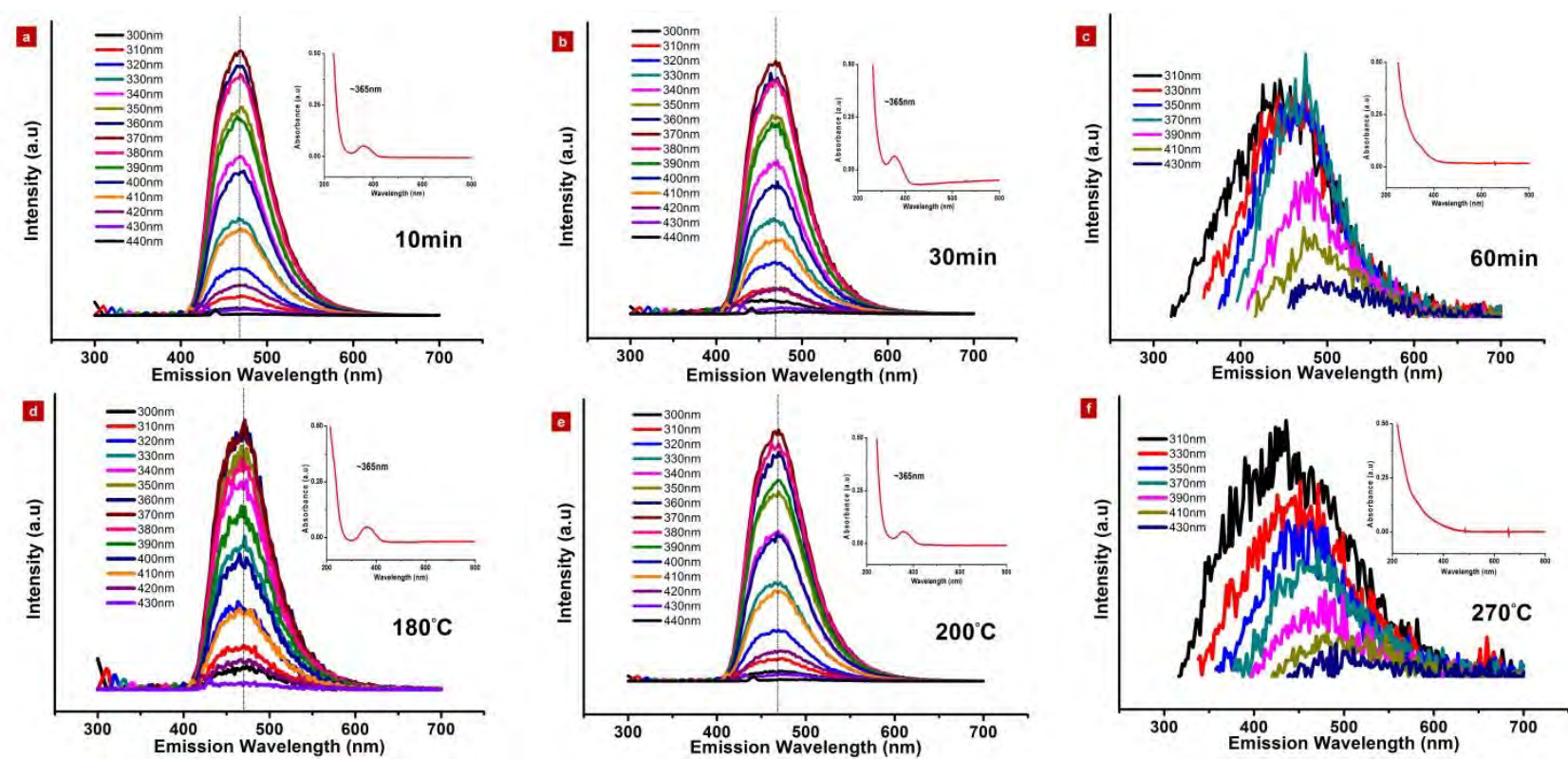

Figure.1 Fluorescence spectroscopies and UV/Vis spectroscopies(inserted) for samples synthesized at $200^{\circ} \mathrm{C}$ for different durations $(\mathrm{a} \sim \mathrm{c})$ and samples synthesized for $20 \mathrm{~min}$ at different temperatures $(d \sim f)$ 


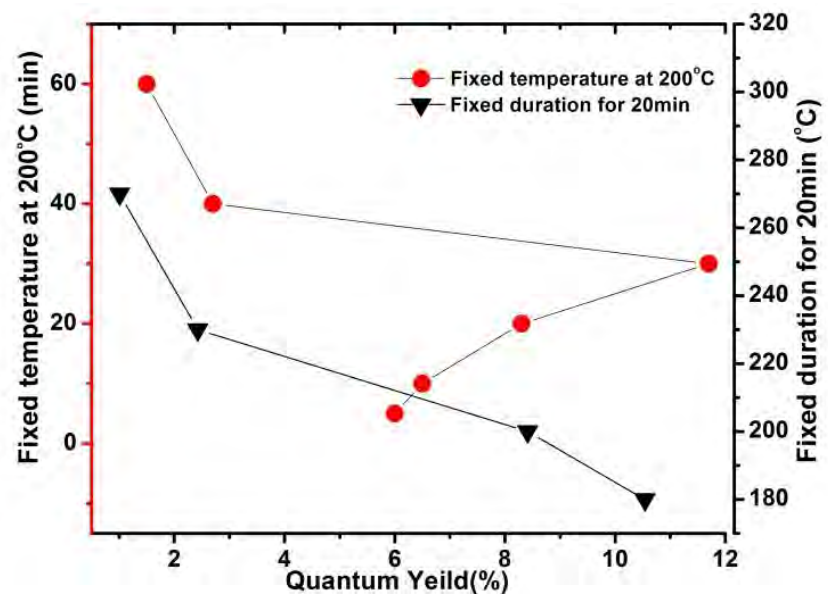

Figure.2 Quantum yields of TDCAs synthesized at fixed temperature $200^{\circ} \mathrm{C}$ and also for fixed duration $20 \mathrm{~min}$

\subsection{Structural evolution during thermolysis}

Both small individual $\mathrm{sp}^{2}$ domains (GQDs) and large sheet-like structures (see supporting information Fig.S2) were obtained in all the TDCA samples synthesized. When the $200^{\circ} \mathrm{C}-30 \mathrm{~min}$ sample was inspected, small particles with an average height around 1.4nm (Fig.3 a) were detected by AFM, however, HRTEM failed to reveal the shapes and sizes of these small particles, which indicates the size of GQDs synthesized at $200^{\circ} \mathrm{C}$ for duration of $30 \mathrm{~min}$ should be either extremely small or instable under electron beam irradiation. Similarly, all GQDs synthesized at $200^{\circ} \mathrm{C}$ with duration shorter than 30min cannot be observed under HRTEM. However, the large particles as the by-product bear close resemblance to GO prepared through oxidation of natural graphite with similar shape and wrinkled surface (Fig.S2). In addition, both AFM and TEM images show obvious crosslinking and stacking during the formation of particles (Fig.3 b and c). Hence, it is highly probable that the reaction mechanism of thermal decomposition of citric acid is that citric acid molecules first condense into $\mathrm{sp}^{2}$ domains forming nucleus, followed by subsequent crosslinking and stacking as the reaction proceeds . 
In contrast to TDCA synthesized at $200^{\circ} \mathrm{C}$ for $30 \mathrm{~min}$, the TDCAs synthesized at $270^{\circ} \mathrm{C}$ for $20 \mathrm{mins}$ contains small particles of size 5 7nm with discernable graphitic domains under HRTEM (Fig.2d). The TEM observation for both samples is consistent with our DLS data. In general, the DLS data show multimodal particle size distributions (Fig.3e). In the sample synthesized at $200^{\circ} \mathrm{C}$ for $30 \mathrm{~min}$ (upper diagram in Fig.3e), DLS detected a small portion of narrow distribution with average size about $0.9 \mathrm{~nm}$ (GQDs) and large particles in the range from $50 \mathrm{~nm}$ to several microns. In the sample synthesized at $270^{\circ} \mathrm{C}$ for $20 \mathrm{~min}$, however, GQDs with an average size around $7 \mathrm{~nm}$ were detected by DLS (lower diagram in Fig.3e).
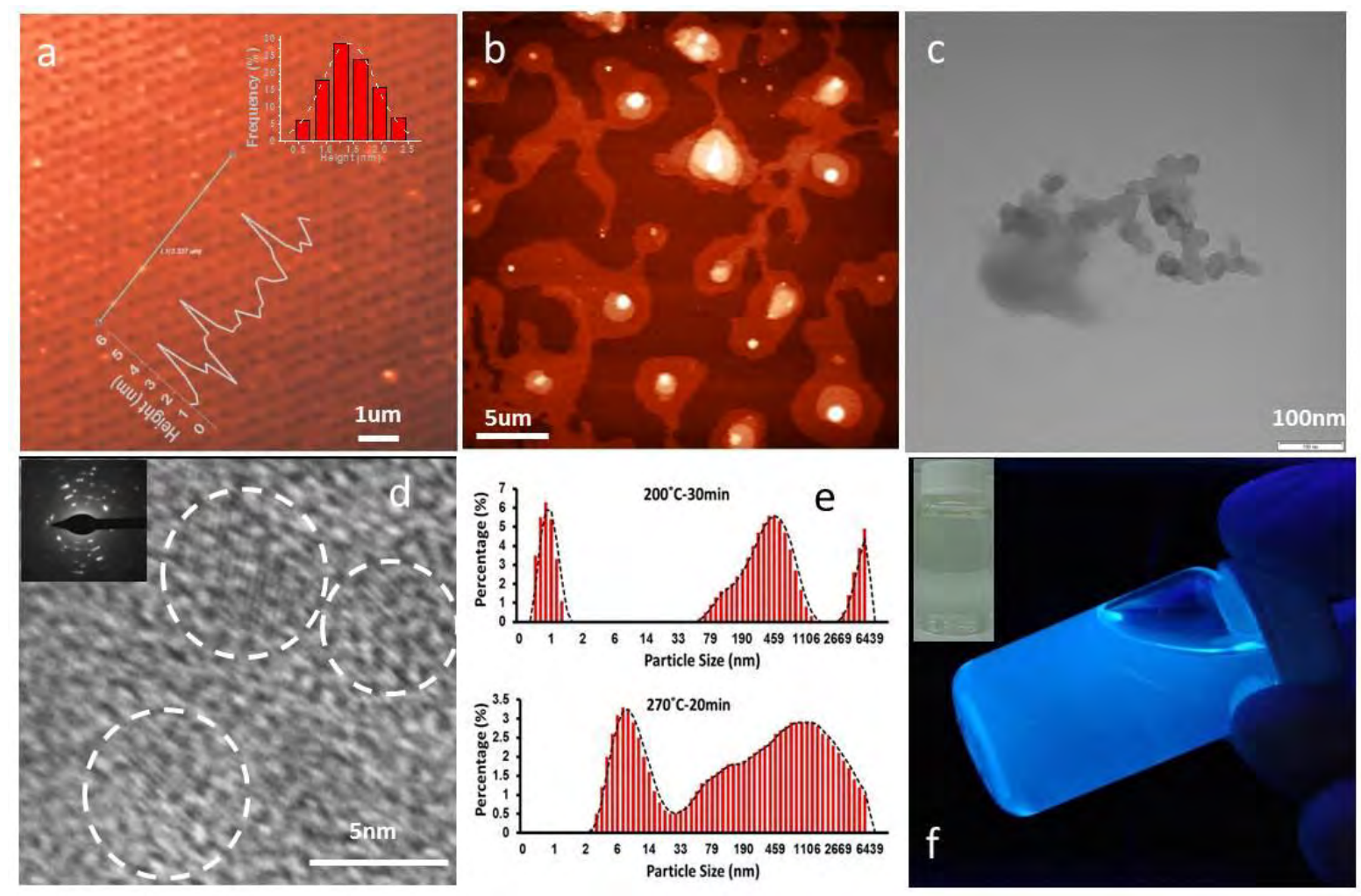

Figure. 3 AFM and TEM images of TDCA sample prepared at $200^{\circ} \mathrm{C}$ for $30 \min (\mathrm{a} \sim \mathrm{c})$ : a, AFM image of GQDs (insets: upper corner to the right is the height profile distribution which was obtained from over 300 GQDs and lower corner to the left is the height profile of three GQDs), b and c, AFM and TEM image showing crosslinking and stacking of TDCAs ; HRTEM image of GQDs prepared at $270^{\circ} \mathrm{C}$ for $20 \mathrm{~min}(\mathrm{~d})$, DLS data for TDCAs prepared at $200^{\circ} \mathrm{C}$ for $30 \mathrm{~min}$ (upper) and $270^{\circ} \mathrm{C}$ for 
$20 \mathrm{~min}$ (lower) (e) and; photo of GQDs $\left(200^{\circ} \mathrm{C}\right.$ for $\left.30 \mathrm{~min}\right)$ dispersion in water under $365 \mathrm{~nm} \mathrm{UV}$ lamp (f, inset:the same dispersion under roomlight).

Secondly, further analysis of the DLS data of all the TDCAs synthesized in our study reveals important information on the population evolution of particles with tunning synthesis conditions. For the samples prepared at the fixed temperature of $200^{\circ} \mathrm{C}$, the percentage of the extremely small GQDs (average size in the range of $0.7 \sim 1 \mathrm{~nm}$ ) contained in the TDCAs increased as reaction proceeded till reaching a peak of $24 \%$ at $30 \mathrm{~min}$, and then dropped accompanied by a rapid percentage rise of much larger-sized particles. Eventually, no GQDs of such small size could be detected after reacting for $60 \mathrm{~min}$ (Fig.4a). On the other hand, for the fixed reaction duration of $20 \mathrm{~min}$, the higher the temperature, the lower the percentage of the tiny GQDs ( average size $0.7 \sim 1 \mathrm{~nm}$ ); typically when temperature is higher than $230^{\circ} \mathrm{C}$, no GQDs in size range of $0.7 \sim 1 \mathrm{~nm}$ could be detected (Fig.4b). This size distribution data are intimately related to the PL evolution trend described earlier in 3.1. Namely, high QY, excitation-independent PL can be observed in the TDCAs containing sufficient GQDs sized $0.7 \sim 1 \mathrm{~nm}$. For the TDCAs without the population of $0.7 \sim 1 \mathrm{~nm}$ particle, they exhibit low QY, and excitation-dependent PL. This observation suggests that the evolution trend of the PL properties of TDCAs with temperature and reaction time is likely due to the competition between the small particles with narrow size range (average size $0.7 \sim 1 \mathrm{~nm}$ ) and large particles of broad size distribution.
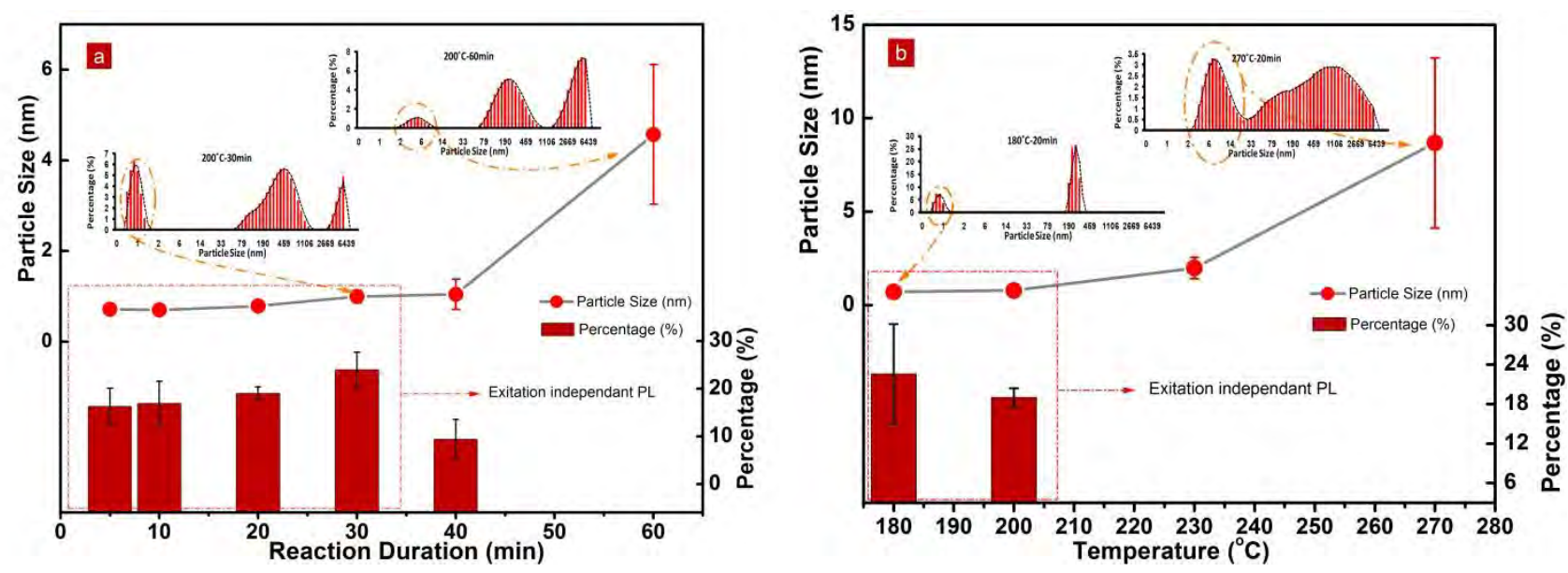
Figure.4 DLS summary on particle size and percentage of small sized GQDs (average particle size $0.7 \sim 1 \mathrm{~nm}$ ) in the TDCAs: a) fixed temperature at $200^{\circ} \mathrm{C}$ and b) fixed duration for $20 \mathrm{~min}$.

According to previous research on the PL of GO [33], both peak wavelength and emission intensity are significantly dominated by the size of the $\mathrm{sp}^{2}$ domains scattered within the basal plane of GO sheets.. GQDs are in fact individual $\mathrm{sp}^{2}$ domains, which also possess size-dependant PL [25, 31]. GQDs made from majority of the top-down methods normally have particle size distribution of wide range, therefore their fluorescence spectra exhibit excitation-dependence. However, in our case, the portion of the small particles which is very uniform in terms of size should be responsible for the excitation-independent high-QY PL of TDCAs, whereas, the portion of the large particles with inhomogeneous sizes should be the source of excitation-dependent low-QY PL of TDCAs. Therefore, it is highly likely that the competition in population between the two parts in the TDCAs determines whether the PL is excitation energy-independent or excitation energy-dependent. To confirm this suggestion, dialysis was performed to partition the samples. Through dialysis $(3.5 \mathrm{kDa})$ treatment, it was clearly observed that the fraction which penetrated the dialysis membrane possesses excitationindependent, intense blue emission similar to the original samples, whereas the fraction remained inside the dialysis membrane tube show apparent excitation-dependant features and the luminescence is much weaker in comparison (Fig.5).

Additionally, Figure 5d shows an obvious trend of increasing QY with reaction time for the $<3.5$ kDa portion of TDCAs. According to the DLS data, the sizes of the GQDs for the listed durations are quite close, therefore this increase of QY of GQDs may be caused by the level of reduction of GQDs 

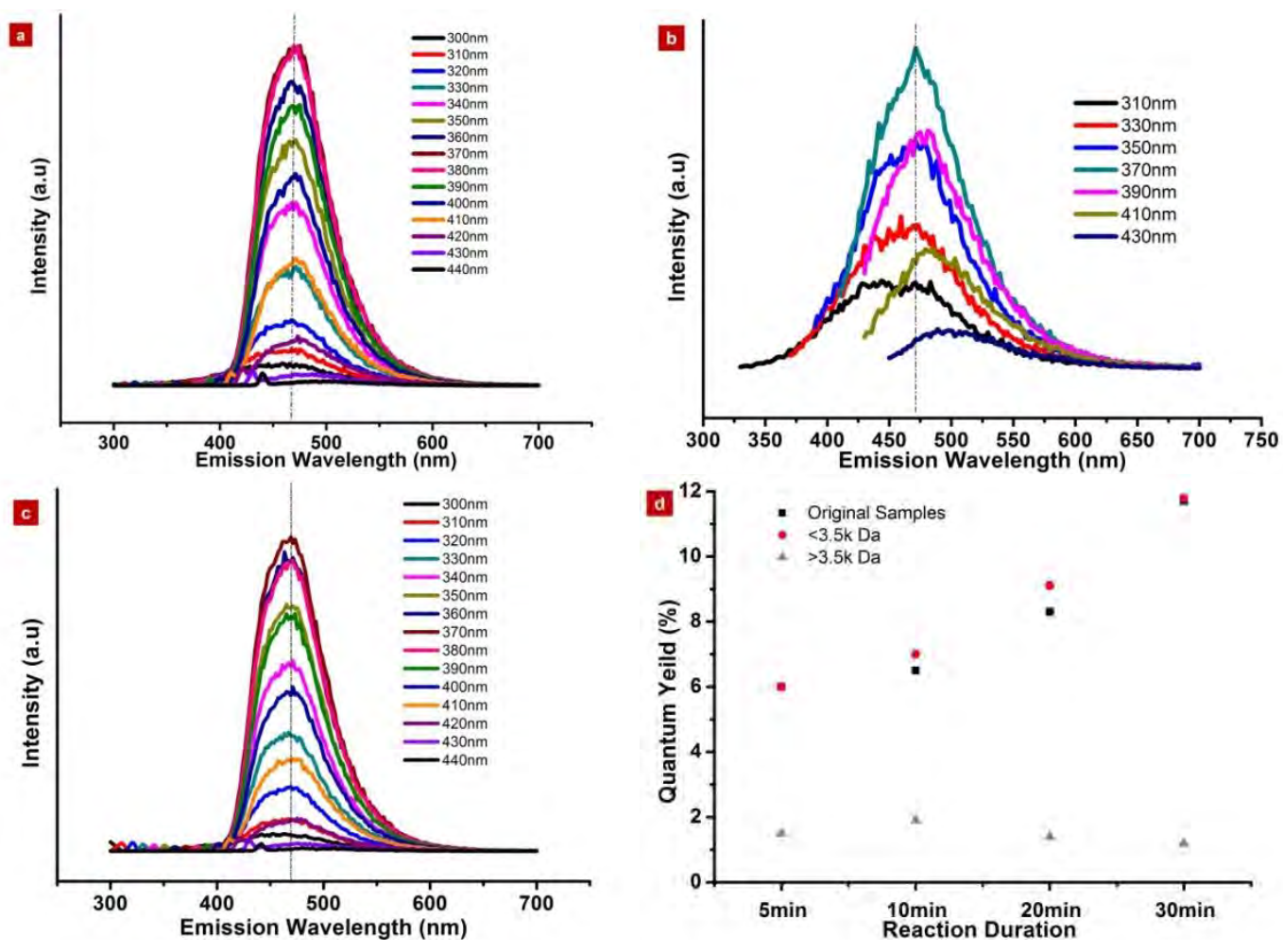

Figure. 5 Fluorescent spectroscopies for TDCA synthesized at $200^{\circ} \mathrm{C}$ for $30 \mathrm{~min}$ with dialysis applied $(a \sim c)$ : a) the section penetrated dialysis membrane, b) the section left inside the dialysis membrane, c) original TDCA, and; quantum yields before (Original samples) and after dialysis $(<3.5 \mathrm{k}$ Da and $>3.5 \mathrm{k}$ Da refers to the section penetrated and the section left inside the dialysis membrane tube respectively) for samples synthesized at $200^{\circ}$ Cfor different durations (d)

as a function of thermolysis duration. Indeed, the carbon-oxygen ratio of GQDs from XPS analysis, decreases with the increase of reaction time (Table.1). This observation shows that as thermolysis proceeds over time, the oxygen content of the GQDs reduces, which resulted in higher QY, consistent with the findings of Zhu et al [20]. The thermally induced reduction in oxygen content can be further specified by analysing the chemical bonds via XPS peak fitting. As shown in Fig. 6, at a fixed temperature of $200^{\circ} \mathrm{C}$, with the extension of reaction duration, the amount of both hydroxyl ($\mathrm{C}-\mathrm{OH})$ and carboxyl groups $(\mathrm{O}=\mathrm{C}-\mathrm{OH})$ decreased, whereas the amount of carbon-carbon bonding $(\mathrm{C} / \mathrm{C}=\mathrm{C})$ increased. Detailed percentage of all observed chemical bonds in the analysed samples were 
provided in Table 1. It should also be noted that there is a gradual increase of carbonyl group content, which can be ascribed to the conversion of carboxyl group possessed by the precursor citric acid.
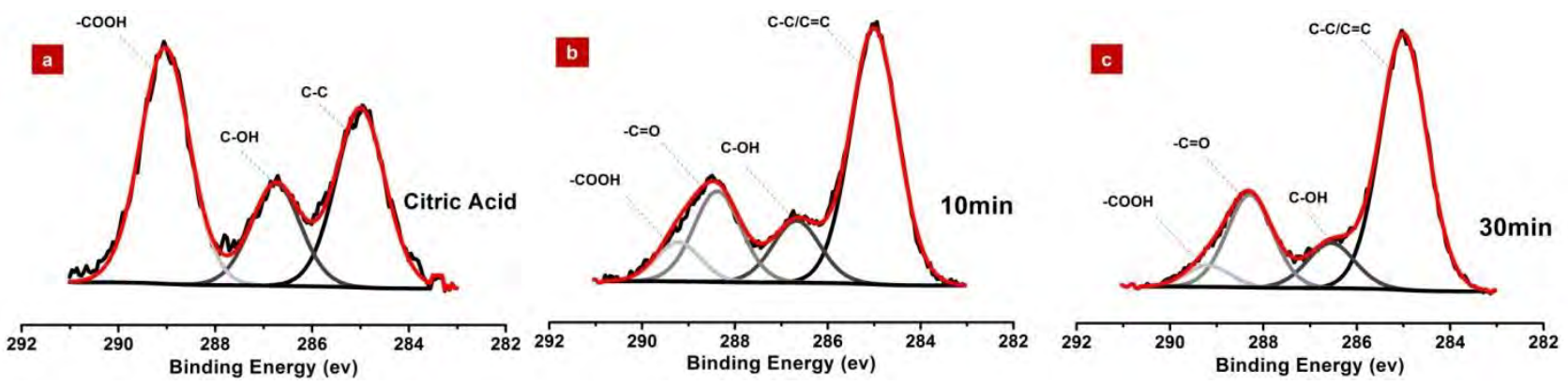

Figure.6 XPS peaks fitting for pure citric acid(a), GQDs $(<3.5 \mathrm{~K} \mathrm{Da})$ prepared at $200^{\circ} \mathrm{C}$ for different durations

\begin{tabular}{ccccccc}
\hline Durations & $\mathrm{C}: \mathrm{O}$ & $\mathrm{C}-\mathrm{C} / \mathrm{C}=\mathrm{C}$ & $\mathrm{C}-\mathrm{O}$ & $\mathrm{O}=\mathrm{C}$ & $\mathrm{COOH}$ & $\mathrm{QY}$ \\
& & $285 \mathrm{ev}$ & $\sim 286.4 \mathrm{ev}$ & $\sim 288.3 \mathrm{ev}$ & $\sim 289.1 \mathrm{ev}$ & \\
\hline 0min & 1.1 & 34.6 & 19.7 & & 45.6 & \\
$5 \mathrm{~min}$ & 1.5 & 50.4 & 15.8 & 19.9 & 13.9 & 6.0 \\
$10 \mathrm{~min}$ & 2.3 & 57.5 & 14.0 & 20.4 & 8.1 & 7.0 \\
$20 \mathrm{~min}$ & 2.4 & 61.9 & 11.6 & 21.3 & 5.2 & 9.1 \\
$30 \mathrm{~min}$ & 2.3 & 62.1 & 11.1 & 21.9 & 4.9 & 11.8 \\
\hline
\end{tabular}

Table.1 C1s bonding states assignment from XPS peak fitting, carbon-oxygen ratio and PL quantum yields for GQDs synthesized at $200^{\circ} \mathrm{C}$ for different durations

For a fixed duration at $20 \mathrm{~min}$, GQDs with sizes $<3.5 \mathrm{kDa}$ could only be obtained at low temperatures $\left(180^{\circ} \mathrm{C}, 200^{\circ} \mathrm{C}\right)$, therefore, the XPS data of as prepared TDCA samples were measured for analysis of chemistry evolution with varying temperatures. As displayed in Figure 7, decrease in hydroxyl and carboxyl groups, and increase of the carbon-carbon bonding $(\mathrm{C} / \mathrm{C}=\mathrm{C})$ is observed (see Table.2 for specific contents of different bonding states), similar to the fixed temperature case. However, the amount of carbonyl groups experienced a decline, which means that higher temperatures could remove the carbonyl groups better than the low temperatures and ensures better crystallization. As 
for the QY of those samples (Table.2), although the degree of reduction is also raised with elevating temperature, it hardly improved the QY of TDCAs because the effect is rapidly overtaken by the percentage increase of large particles. Therefore, a rapid diminishing trend of QY with temperature rise was observed.
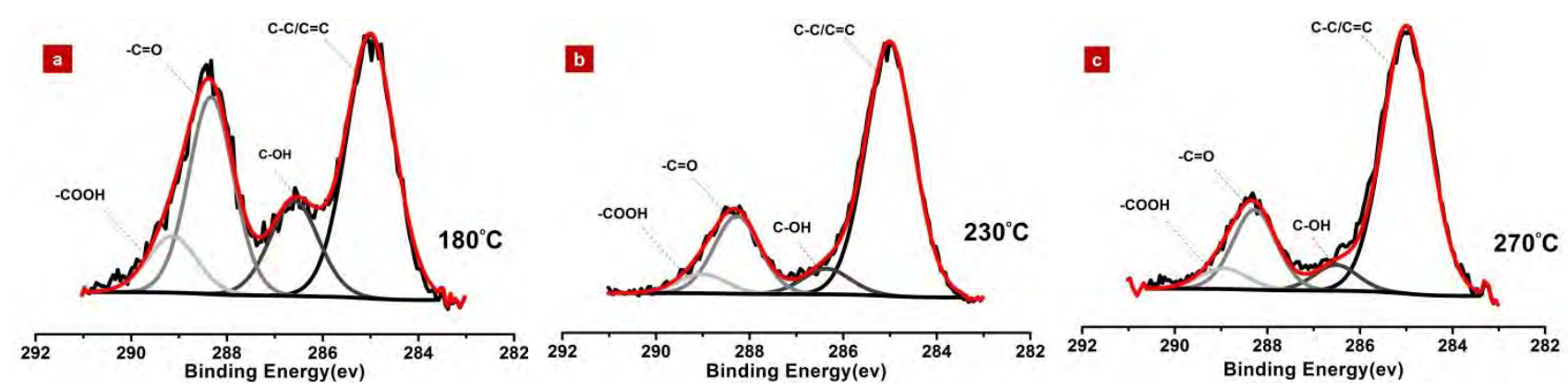

Figure.7 XPS peaks fitting for TDCAs prepared at different temperatures for fixed duration at 20min

\begin{tabular}{ccccccc}
\hline Temperatures & $\mathrm{C}: \mathbf{O}$ & $\begin{array}{c}\mathrm{C}-\mathrm{C} / \mathrm{C}=\mathrm{C} \\
\sim \mathbf{2 8 5 e v}\end{array}$ & $\begin{array}{c}\mathrm{C}-\mathrm{O} \\
\sim \mathbf{2 8 6 . 4 e v}\end{array}$ & $\begin{array}{c}\text { O=C } \\
\sim 288.3 e v\end{array}$ & $\begin{array}{c}\text { COOH } \\
\sim \mathbf{2 8 9 . 1 e v}\end{array}$ & QY \\
\hline $\mathbf{1 8 0 ^ { \circ } \mathrm { C }}$ & 1.8 & 44.1 & 15.9 & 30.5 & 9.5 & 10.4 \\
$\mathbf{2 3 0 ^ { \circ } \mathrm { C }}$ & 2.5 & 68.0 & 6.9 & 20.0 & 5.2 & 2.4 \\
$\mathbf{2 7 0 ^ { \circ } \mathrm { C }}$ & 2.9 & 69.5 & 6.2 & 19.0 & 5.4 & 1.0
\end{tabular}

Table.2 C1s bonding states assignment from XPS peak fitting, carbon-oxygen ratio and PL quantum yields for TDCAs synthesized for 20min at different temperatures

From the evolution trend of the PL properties as a function of the structure of TDCAs, a clear understanding could be obtained on the thermal decomposition process, as illustrated in Figure.8. Firstly, higher temperature results in fiercer reactions.; it takes less time for GQDs with average size in the range of $0.7 \sim 1 \mathrm{~nm}$ to nucleate from citric acid molecules and then crosslink and stack to form larger particles. At higher temperature, most of the tiny GQDs would be consumed very quickly to form particles with larger inhomogeneous sizes than in the case of lower temperatures. Therefore, the excitation-dependant, low QY emission from the larger-sized particles dominates the PL of TDCAs. 
On the other hand, for a fixed temperature, with the extension of reaction time, more citric acid molecules will be consumed to form GQDs, meanwhile GQDs will crosslink and stack to form larger particles. The longer the duration, more large particles will be constructed, eventually with few tiny GQDs which possess excitation-independent intense PL left inside the TDCAs, hence the excitationdependant low QY emission from the larger-inhomogenesously-sized particles becomes dominant. Only for those cases at relatively lower temperatures $\left(180^{\circ} \mathrm{C}, 200^{\circ} \mathrm{C}\right)$ and for relatively shorter durations, there are sufficient quantities of highly luminescent, uniformly-sized GQDs within the TDCAs which therefore exhibit strong excitation-independent PL.

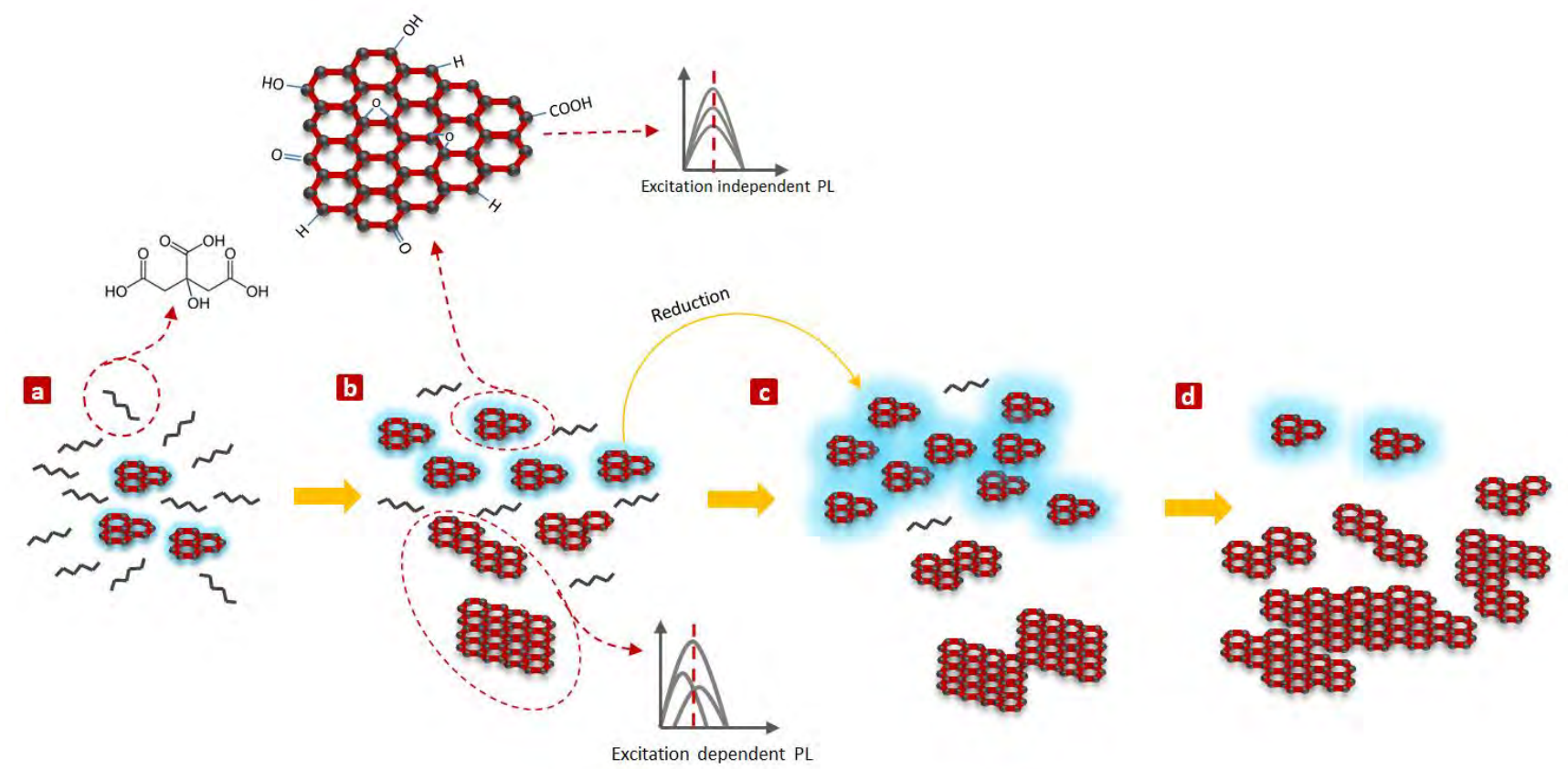

Figure.8 Schematic representation of the thermal decomposition process of CA: a, GQDs nucleate from CA molecules; $b$, further GQDs nucleation with more CA molecules consumed, meanwhile GQDs crosslinking and stacking to form large particles with inhomogeneous sizes which possess excitation dependant PL; c, when the amount of GQDs reaches maximum value, PL of the thermal decomposed product reached optimal level with highest QY; d, with extension of reaction duration, most of the GQDs are consumed for formation of large particles, therefore, excitation-dependant PL of those large particles with ununiformed sizes dominates the PL of TDCAs. From a $\sim$, GQDs are 
also reduced to a lower oxygen content levels, which in principle improves the PL of the thermal decomposition process.

\subsection{Cation sensing}

As shown in the XPS study, the GQDs possess abundant negative charged surface moieties, such as hydroxyl and carboxyl groups. The interaction between these functional groups and cations may influence the PL of GQDs, which can be used for sensor development. Figure 9a shows the PL intensity of GQDs solutions synthesized at $200^{\circ} \mathrm{C}$ for $30 \mathrm{~min}$ with different cations of the same loading $0.25 \mathrm{mM} / \mathrm{L}$. It is apparently that all tested cations possess certain degree of quenching effect on PL of GQDs, however, $\mathrm{Fe}^{3+}$ has the most significant quenching efficiency than the rest, which means that raw GQDs have excellent selectivity towards $\mathrm{Fe}^{3+}$. Figure $9 \mathrm{~b}$ presents the correlation between $\mathrm{Fe}^{3+}$ concentration and PL of GQDs dispersions. A fine linear relationship between $\mathrm{Fe}^{3+}$ concentration and PL intensity of GQDs dispersions could be constructed.
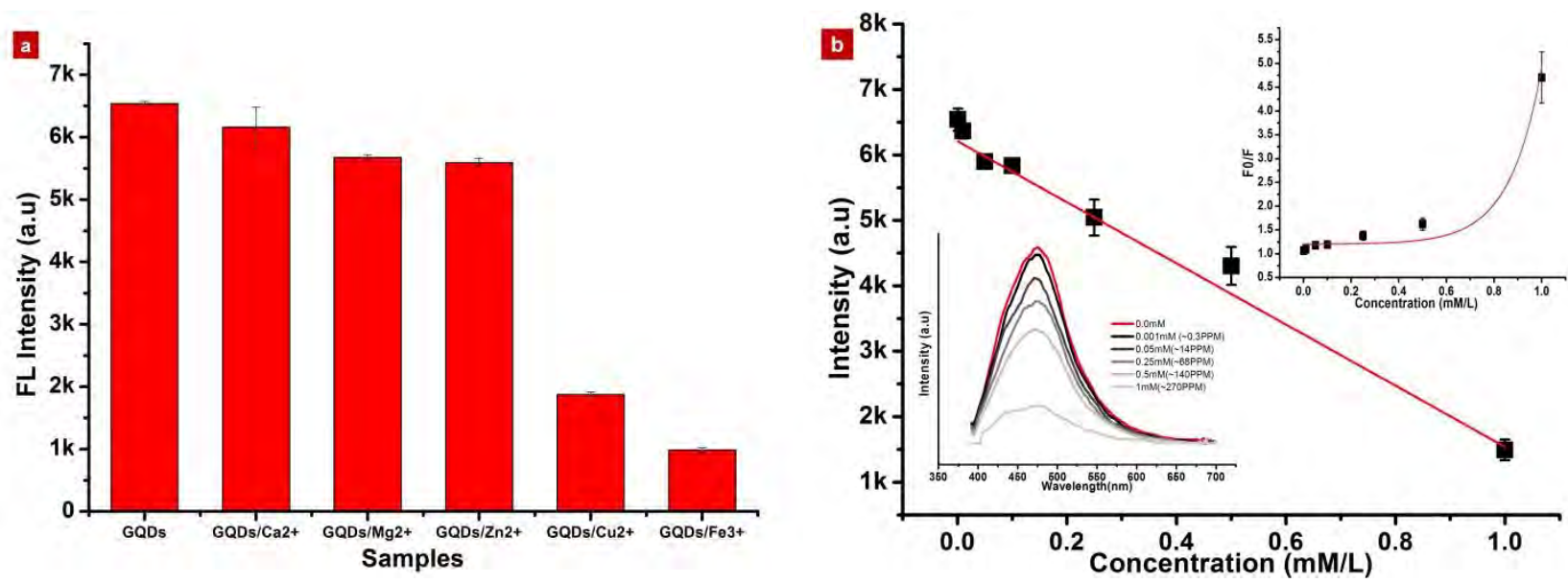

Figure.9 $\mathrm{Fe}^{3+}$ sensing illustration: a, Quenching capability of different cations on PL of GQDs; b, linear relationship between $\mathrm{Fe}^{3+}$ concentration and PL intensity of GQDs. Inserted upper corner to the right is the Stern-Volmer plot and lower corner to the left is the fluorescence spectroscopies of GQDs solutions with different $\mathrm{Fe}^{3+}$ concentration 
To understand the quenching mechanism of the $\mathrm{Fe}^{3+}$ ions quenching of GQDs PL, The Stern-volmer plot was constructed (Inserted figure in Fig.8b at the upper corner to the right), showing a curve shape. This indicates that a combined quenching mechanism (both static and collisional) [34] is responsible for the PL quenching. As shown in Figure 10b, static quenching effect of $\mathrm{Fe}^{3+}$ on the PL of GQDs involves the formation of nonradiative complex ground states between GQDs and $\mathrm{Fe}^{3+}$ through ionic bonding, which results in nonradiative decay upon absorption of incident photons. Therefore, the PL intensity of GQDs is quenched. Whereas, in collisional quenching, upon absorption of photons, electrons are promoted from ground states to exited states in GQDs; however, when electrons of GQDs return to the ground state of GQDs, instead of releasing energy through photons, the energy is absorbed by $\mathrm{Fe}^{3+}$, as shown in Figure 10c. Observable PL quenching could be obtained down to a $\mathrm{Fe}^{3+}$ concentration of $0.3 \mathrm{ppm}$ (Inserted figure in Fig.9b at the lower corner to the left) that illustrates a high sensitivity. Thus, raw GQDs could be used as a sensing material for $\mathrm{Fe}^{3+}$ with excellent both selectivity and sensitivity. Additionally, a point worth mentioning here is that although raw GQDs possess good selectivity and sensitivity to $\mathrm{Fe}^{3+}$ as proved in our research, it is believed that through surface functionalization, raw GQDs synthesized in this investigation could very likely be engineered to fit sensing purposes for not only other cations but also organic chemicals, which is currently under investigation. 


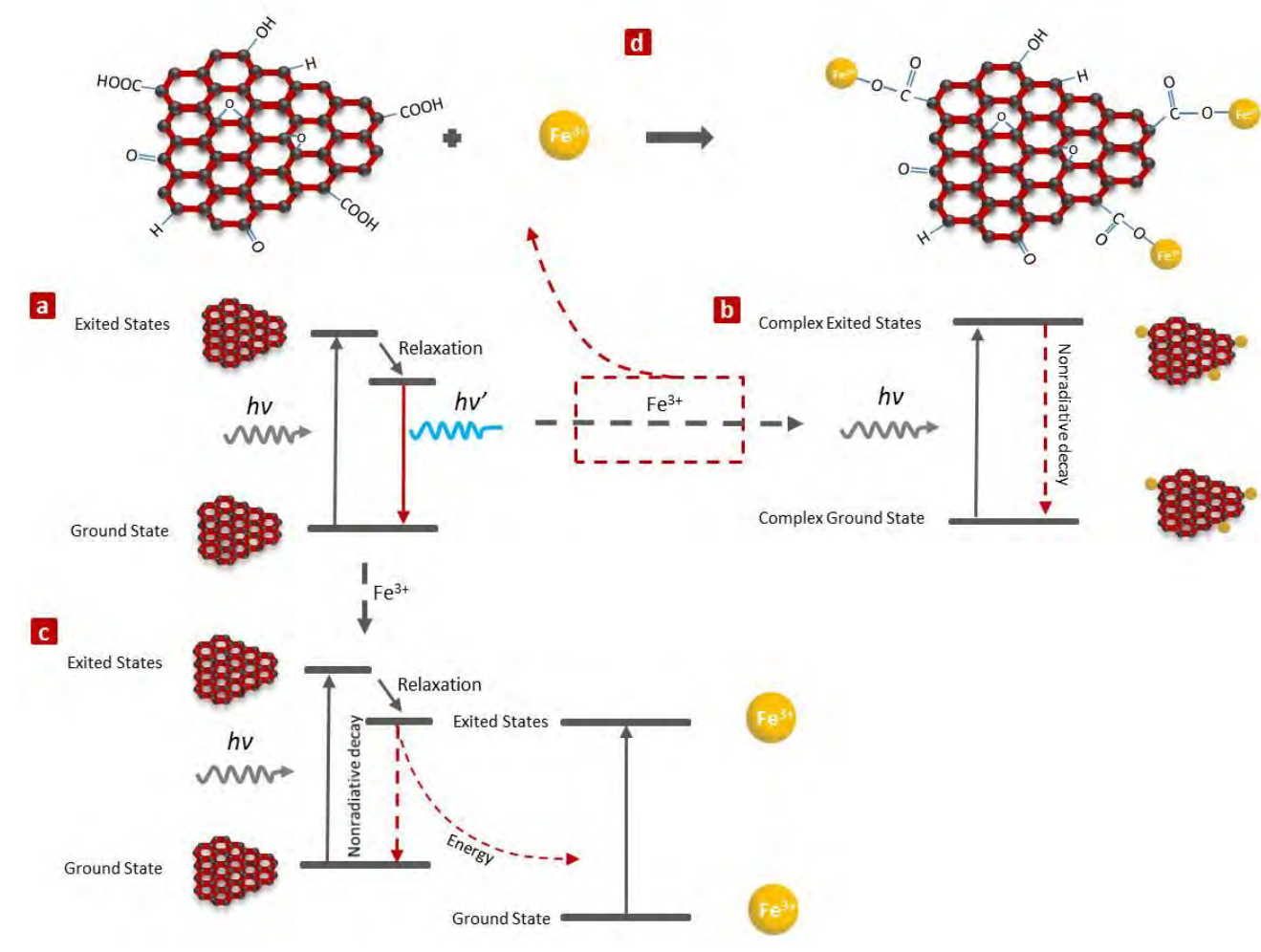

Figure.10 Schematic representation of $\mathrm{Fe}^{3+}$ quenching mechanisms on PL of GQDs: a, natural emission of raw GQDs upon absorption of incident photons; $b$, static quenching effect of $\mathrm{Fe}^{3+}$ on PL of GQDs; c, collisional quenching effects of $\mathrm{Fe}^{3+}$ on PL of GQDs; $\mathrm{d}$, the possible formation mechanism of complex between GQDs and $\mathrm{Fe}^{3+}$ via ionic bonding which is a result of negative charging of raw GQDs.

\section{Conclusion}

By tunning the synthesis conditions, the TDCAs exhibit either excitation-independent or excitationdependant fluorescence spectra and varied QYs. These features are majorly determined by the quantitative competition between the GQDs and the large-sized GO particles in the decomposed products. The fraction of small uniformly sized GQDs (average size $0.7 \mathrm{~nm} \sim 1 \mathrm{~nm}$ ) is responsible for the excitation-independent, high QY blue fluorescence of TDCAs; whereas the fraction of large inhomogeneously sized sheet structures dominate excitation dependent low quantum yield fluorescent emission of TDCAs. When sufficient small GQDs obtained in the TDCAs, they present 
high QY, excitation-independent photoluminescence, otherwise only low QY, excitation-dependent photoluminescence can be seen with the overall product, TDCAs. In addition to addressing the causes behind the trends of PL properties of TDCAs, GQDs' potential as a type of sensing materials for cations were also explored. The raw GQDs synthesized in this study were proved to be an excellent fluorescence sensor for trivalent iron cation with good selectivity and a high sensitivity down to $0.3 \mathrm{ppm}$.

\section{Acknowledgement}

This study was supported by a Griffith University International Postgraduate Scholarship and a CSIRO OCE top-up scholarship.

\section{Reference}

1. Ponomarenko, L.A., et al., Chaotic Dirac billiard in graphene quantum dots. Science, 2008. 320(5874): p. 356-8.

2. Novoselov, K.S., et al., Electric field effect in atomically thin carbon films. Science, 2004. 306(5696): p. 666-9.

3. Barreiro, A., H.S. van der Zant, and L.M. Vandersypen, Quantum dots at room temperature carved out from few-layer graphene. Nano Lett, 2012. 12(12): p. 6096-100.

4. Mandal, B., S. Sarkar, and P. Sarkar, Exploring the electronic structure of graphene quantum dots. Journal of Nanoparticle Research, 2012. 14(12).

5. Yan, X., et al., Independent Tuning of the Band Gap and Redox Potential of Graphene Quantum Dots. The Journal of Physical Chemistry Letters, 2011. 2(10): p. 1119-1124.

6. Agapito, L.A., N. Kioussis, and E. Kaxiras, Electric-field control of magnetism in graphene quantum dots: Ab initio calculations. Physical Review B, 2010. 82(20).

7. Espinosa-Ortega, T., I.A. Luk'yanchuk, and Y.G. Rubo, Magnetic properties of graphene quantum dots. Physical Review B, 2013. 87(20).

8. Ma, W.-L. and S.-S. Li, Electric-field-induced spin depolarization in graphene quantum dots. Physical Review B, 2012. 86(4). 
9. Pan, D., et al., Hydrothermal route for cutting graphene sheets into blue-luminescent graphene quantum dots. Adv Mater, 2010. 22(6): p. 734-8.

10. Ezawa, M., Quasi-ferromagnet spintronics in the graphene nanodisc-lead system. New Journal of Physics, 2009. 11(9): p. 095005.

11. Yan, X., et al., Large, solution-processable graphene quantum dots as light absorbers for photovoltaics. Nano Lett, 2010. 10(5): p. 1869-73.

12. Kou, L., et al., Synthesis of blue light-emitting graphene quantum dots and their application in flexible nonvolatile memory. Organic Electronics, 2013. 14(6): p. 1447-1451.

13. Shen, J., et al., Graphene quantum dots: emergent nanolights for bioimaging, sensors, catalysis and photovoltaic devices. Chem Commun (Camb), 2012. 48(31): p. 3686-99.

14. Rozhkov, A.V., et al., Electronic properties of mesoscopic graphene structures: Charge confinement and control of spin and charge transport. Physics Reports, 2011. 503(2-3): p. 77-114.

15. Girit, C.O., et al., Graphene at the edge: stability and dynamics. Science, 2009. 323(5922): p. 1705-8.

16. Sun, Y., et al., Large scale preparation of graphene quantum dots from graphite with tunable fluorescence properties. Phys Chem Chem Phys, 2013. 15(24): p. 9907-13.

17. Pan, D., et al., Cutting sp2 clusters in graphene sheets into colloidal graphene quantum dots with strong green fluorescence. Journal of Materials Chemistry, 2012. 22(8): p. 3314.

18. Shen, J., et al., One-pot hydrothermal synthesis of graphene quantum dots surface-passivated by polyethylene glycol and their photoelectric conversion under near-infrared light. New Journal of Chemistry, 2012. 36(1): p. 97.

19. Zhu, S., et al., Strongly green-photoluminescent graphene quantum dots for bioimaging applications. Chem Commun (Camb), 2011. 47(24): p. 6858-60.

20. Zhu, S., et al., Graphene quantum dots with controllable surface oxidation, tunable fluorescence and up-conversion emission. RSC Advances, 2012. 2(7): p. 2717.

21. Li, L.-L., et al., A Facile Microwave Avenue to Electrochemiluminescent Two-Color Graphene Quantum Dots. Advanced Functional Materials, 2012. 22(14): p. 2971-2979.

22. Li, Y., et al., An electrochemical avenue to green-luminescent graphene quantum dots as potential electron-acceptors for photovoltaics. Adv Mater, 2011. 23(6): p. 776-80.

23. Shinde, D.B. and V.K. Pillai, Electrochemical preparation of luminescent graphene quantum dots from multiwalled carbon nanotubes. Chemistry, 2012. 18(39): p. 12522-8.

24. Zhang, M., et al., Facile synthesis of water-soluble, highly fluorescent graphene quantum dots as a robust biological label for stem cells. Journal of Materials Chemistry, 2012. 22(15): p. 7461.

25. Peng, J., et al., Graphene quantum dots derived from carbon fibers. Nano Lett, 2012. 12(2): p. 844-9.

26. Minati, L., et al., Luminescent graphene quantum dots from oxidized multi-walled carbon nanotubes. Materials Chemistry and Physics, 2012. 137(1): p. 12-16.

27. Liu, F., et al., Facile synthetic method for pristine graphene quantum dots and graphene oxide quantum dots: origin of blue and green luminescence. Adv Mater, 2013. 25(27): p. 3657-62.

28. Lu, J., et al., Transforming C-60 molecules into graphene quantum dots. Nature Nanotechnology, 2011. 6(4): p. 247-252.

29. Yan, X., X. Cui, and L.S. Li, Synthesis of Large, Stable Colloidal Graphene Quantum Dots with Tunable Size. Journal of the American Chemical Society, 2010. 132(17): p. 5944-+.

30. Liu, R., et al., Bottom-up fabrication of photoluminescent graphene quantum dots with uniform morphology. J Am Chem Soc, 2011. 133(39): p. 15221-3.

31. Tang, L., et al., Size-Dependent Structural and Optical Characteristics of Glucose-Derived Graphene Quantum Dots. Particle \& Particle Systems Characterization, 2013. 30(6): p. 523-531.

32. Dong, Y., et al., Blue luminescent graphene quantum dots and graphene oxide prepared by tuning the carbonization degree of citric acid. Carbon, 2012. 50(12): p. 4738-4743.

33. Eda, G., et al., Blue photoluminescence from chemically derived graphene oxide. Adv Mater, 2010. 22(4): p. 505-9.

34. Lakowicz, J.R., Principles of fluorescence spectroscopy. 1983, New York: Plenum Press. xiv, 496 p. 


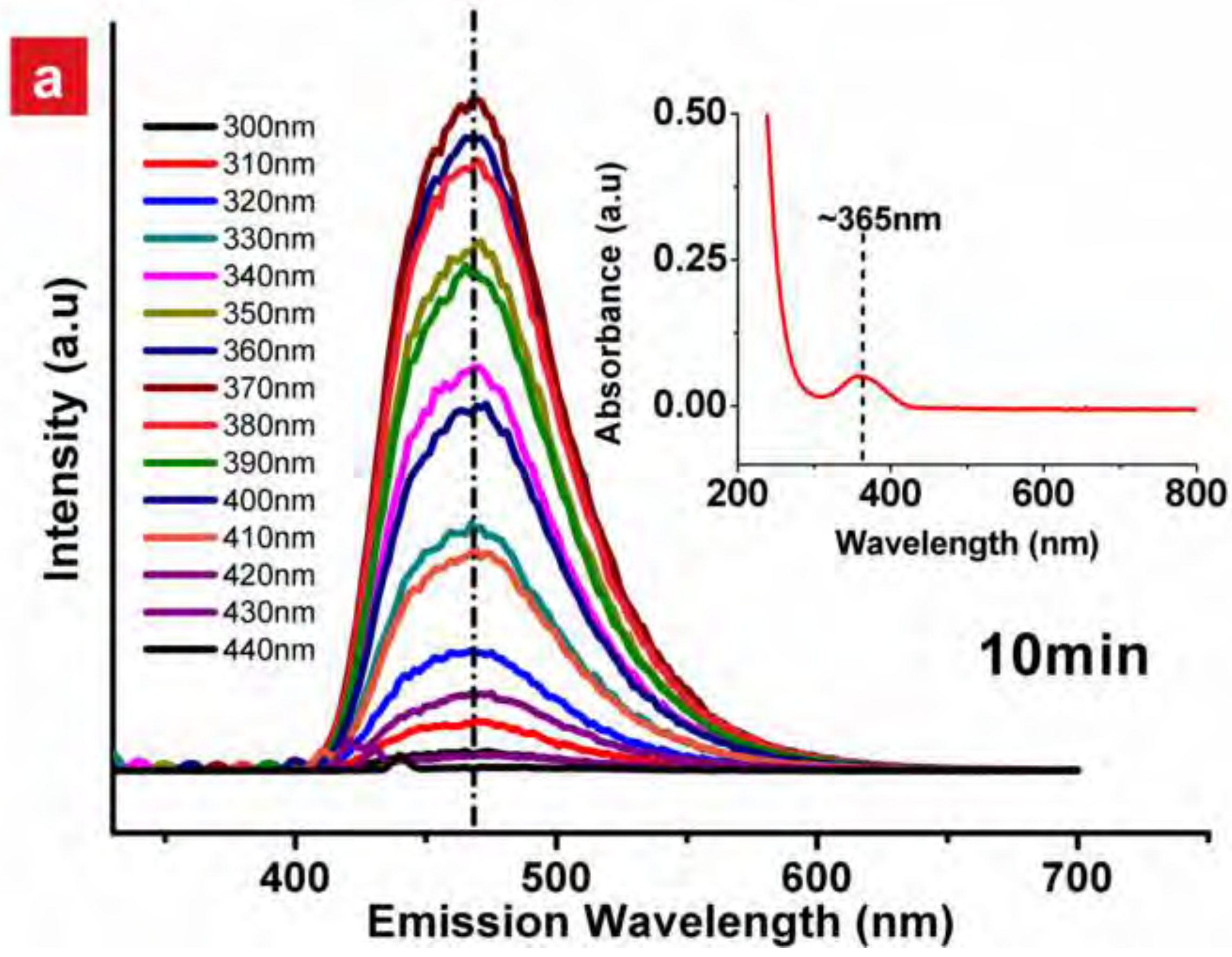




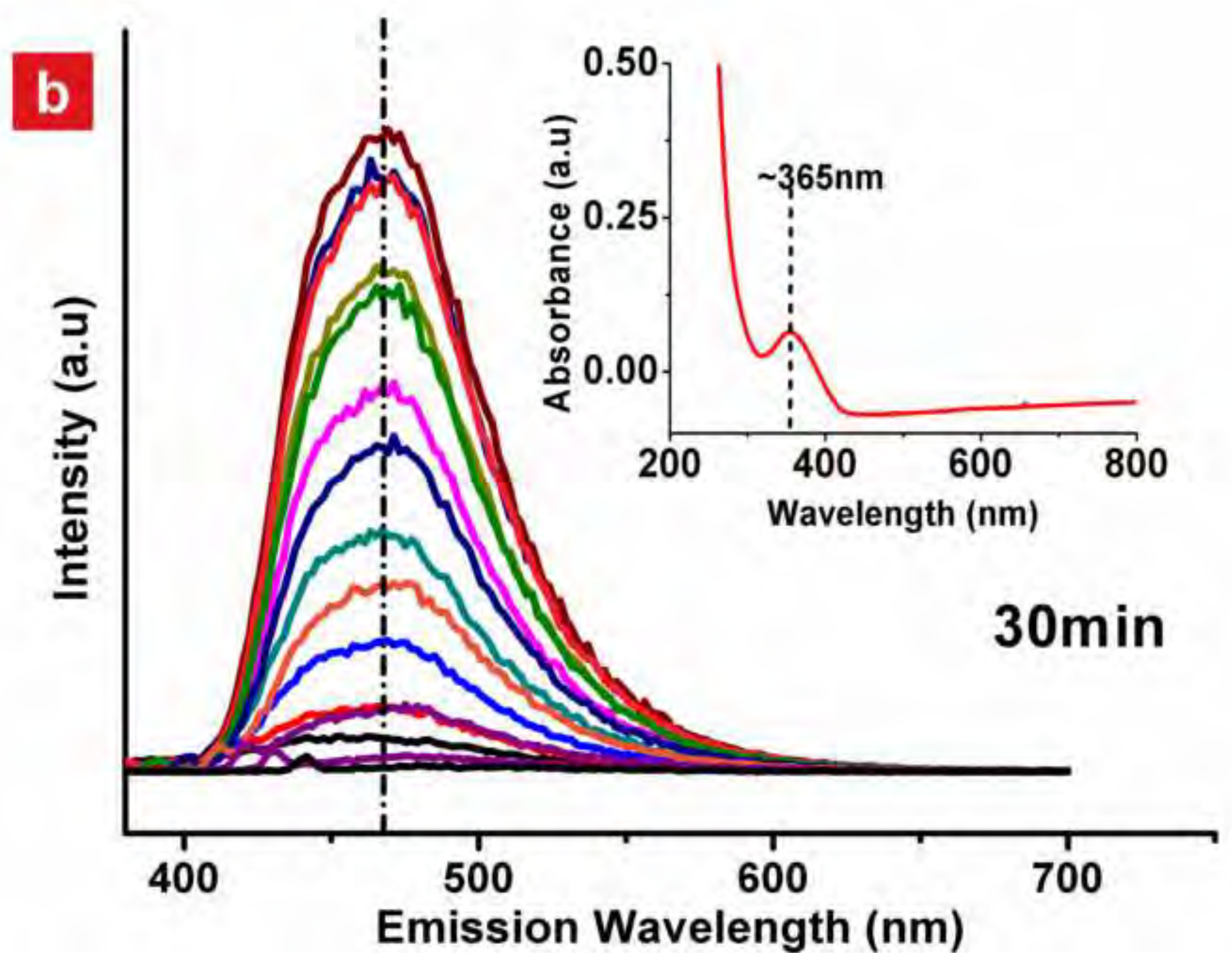




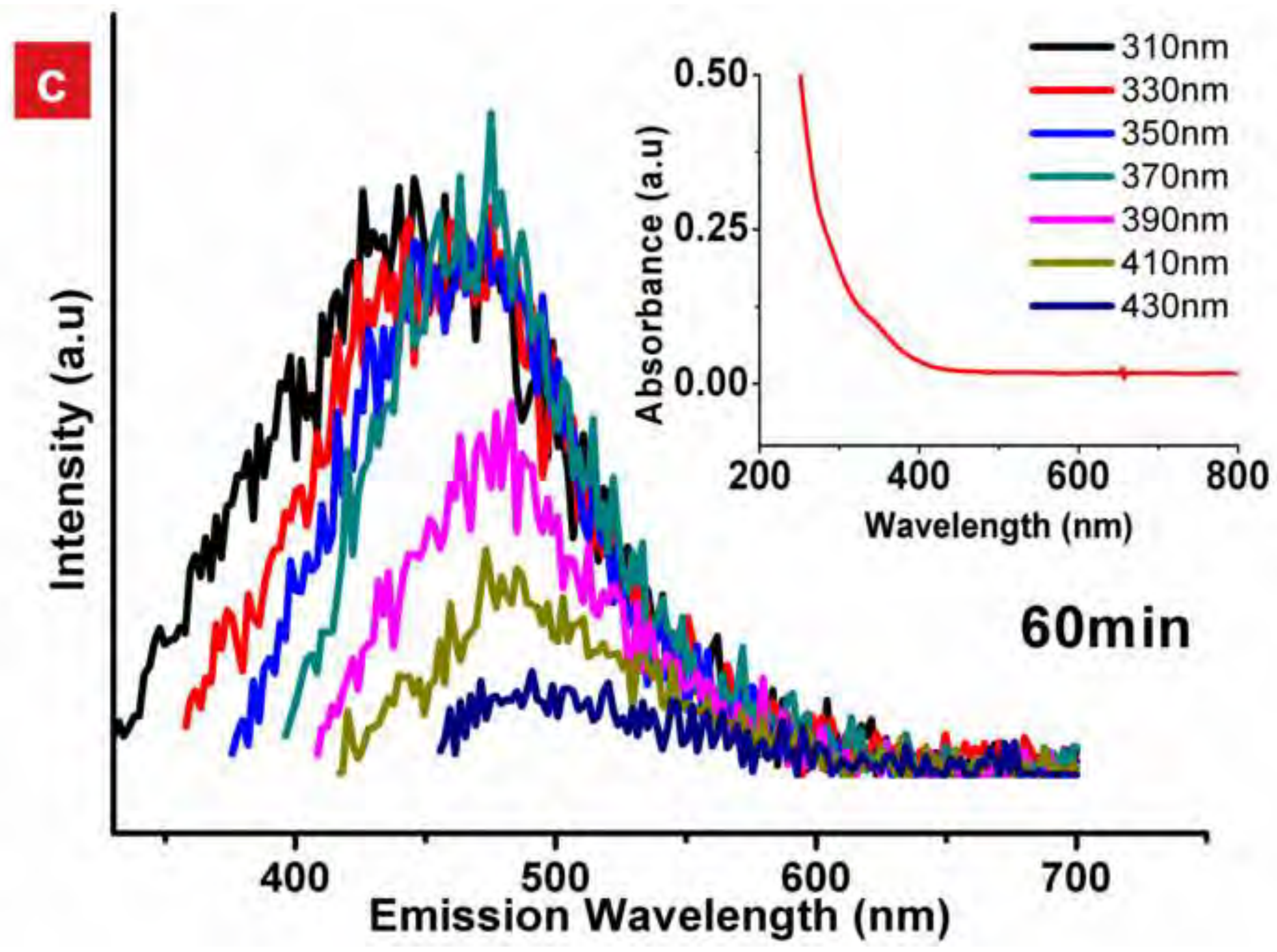




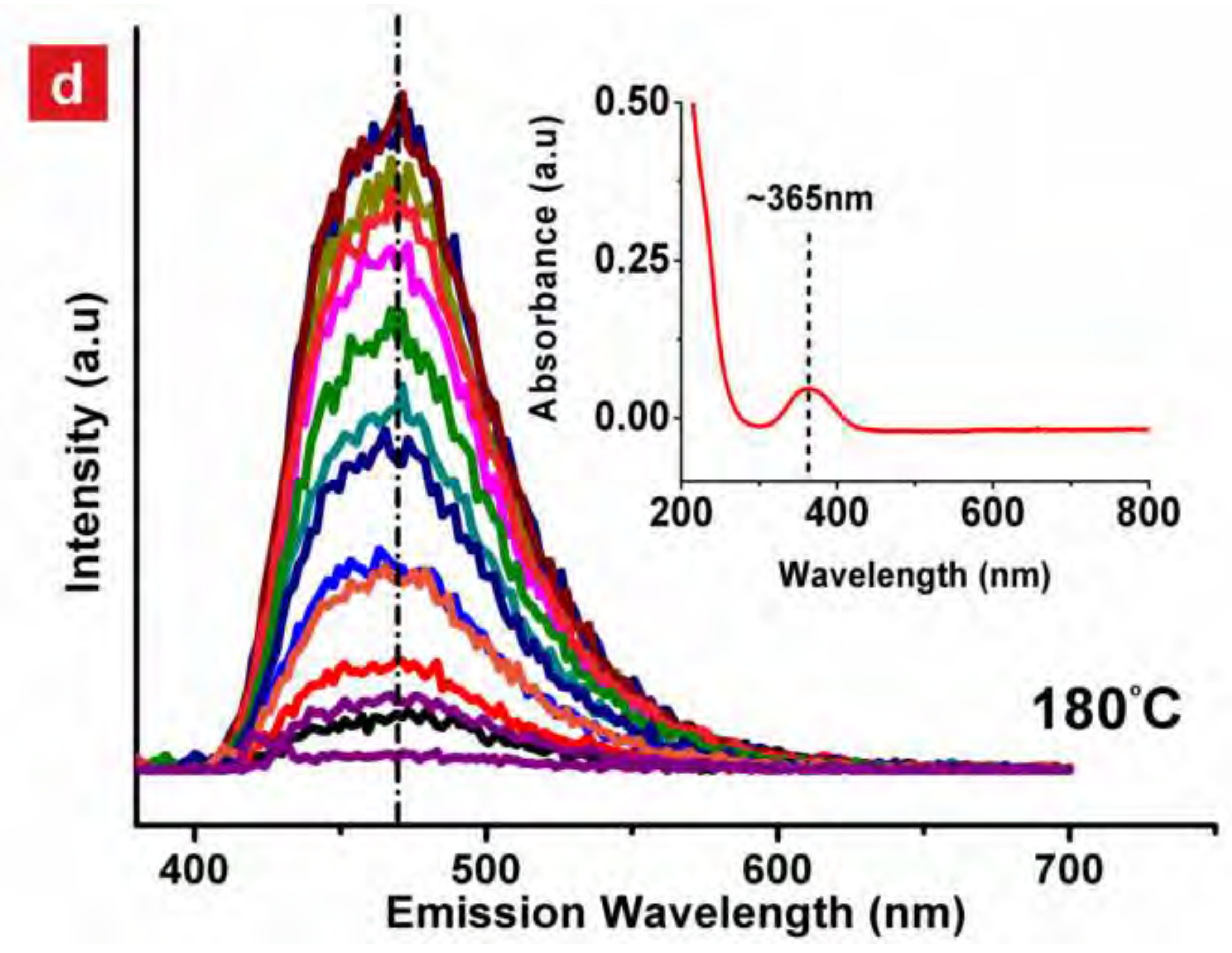




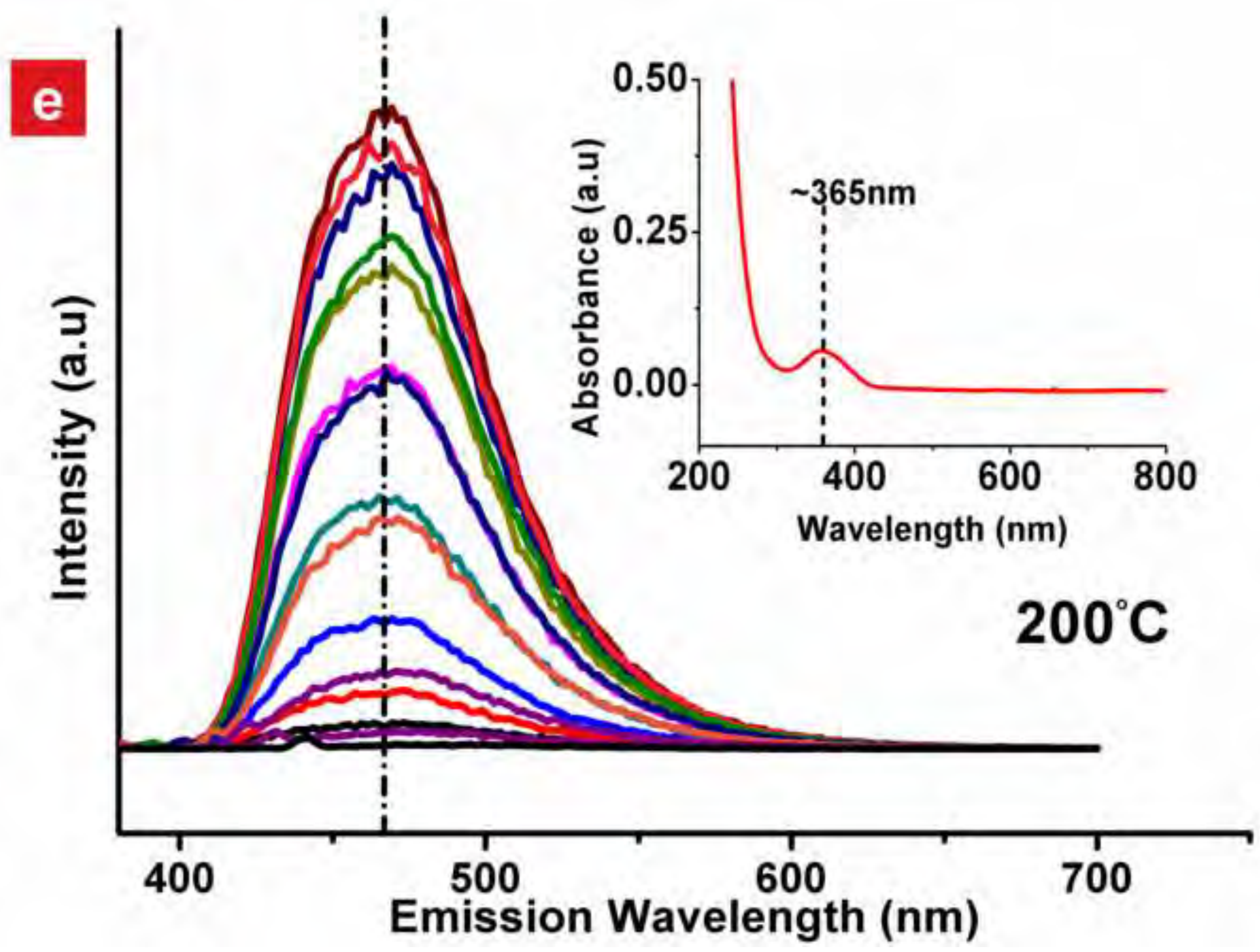




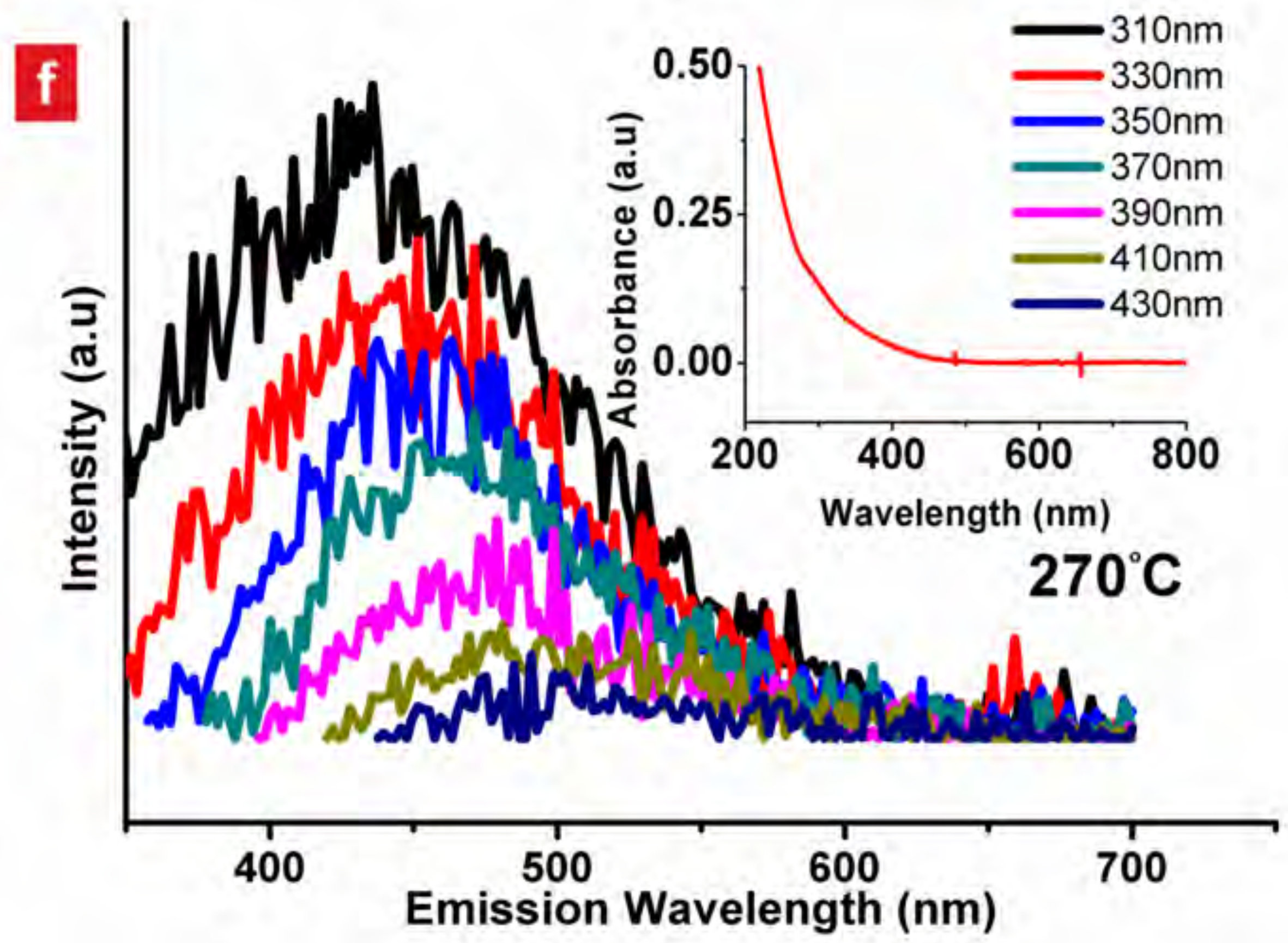


Figure 2

Click here to download high resolution image

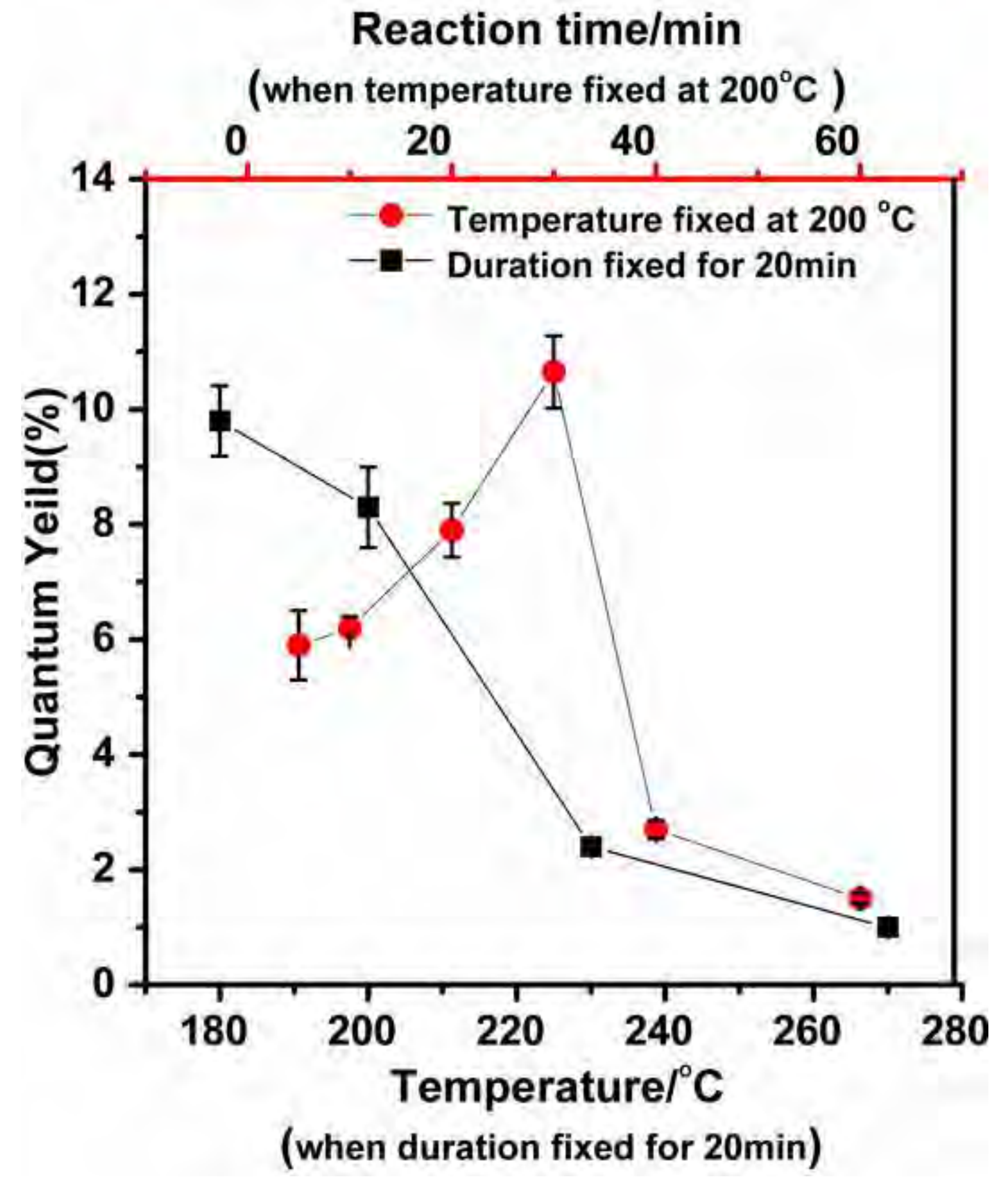

Reaction time/min 

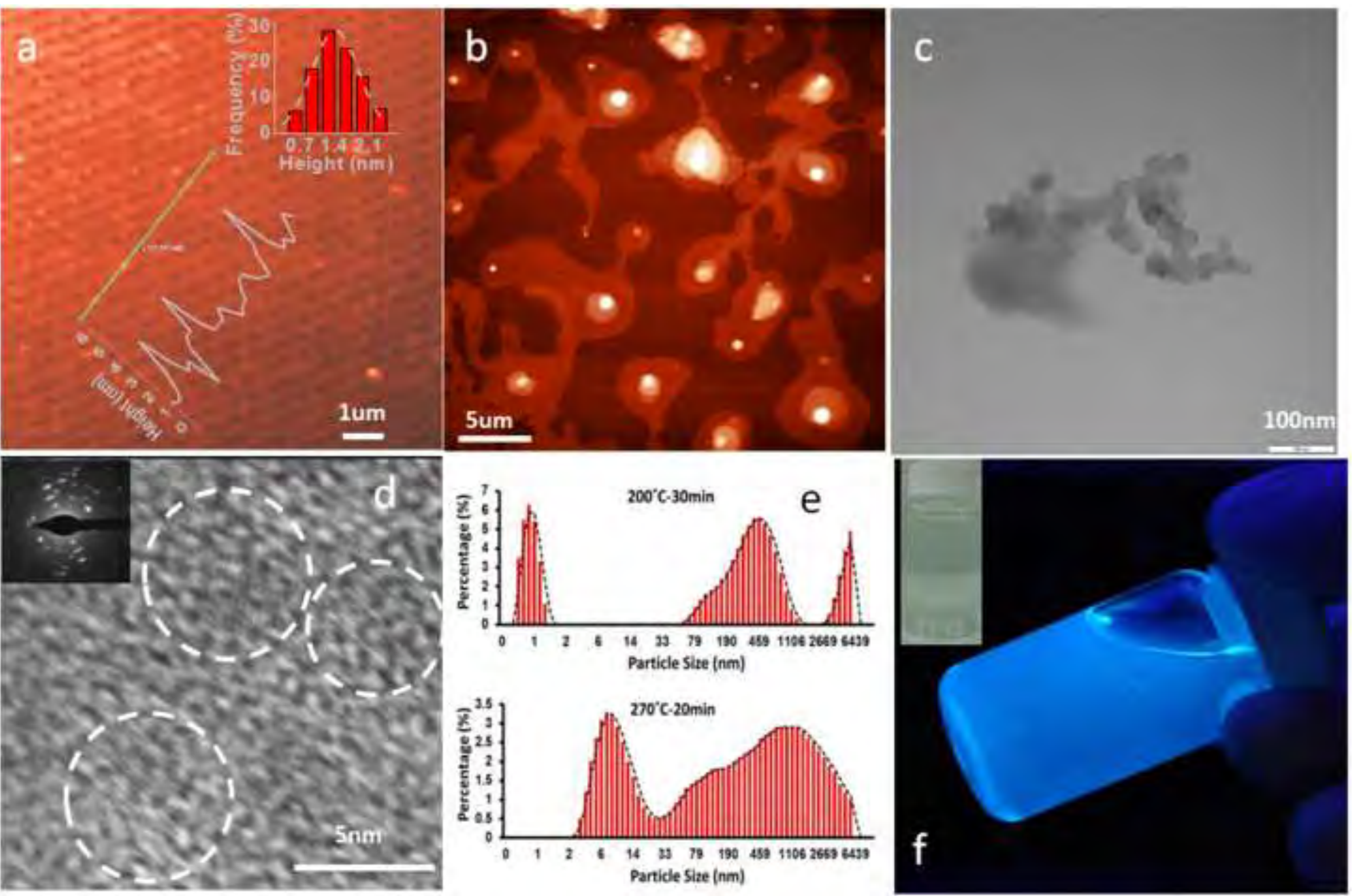


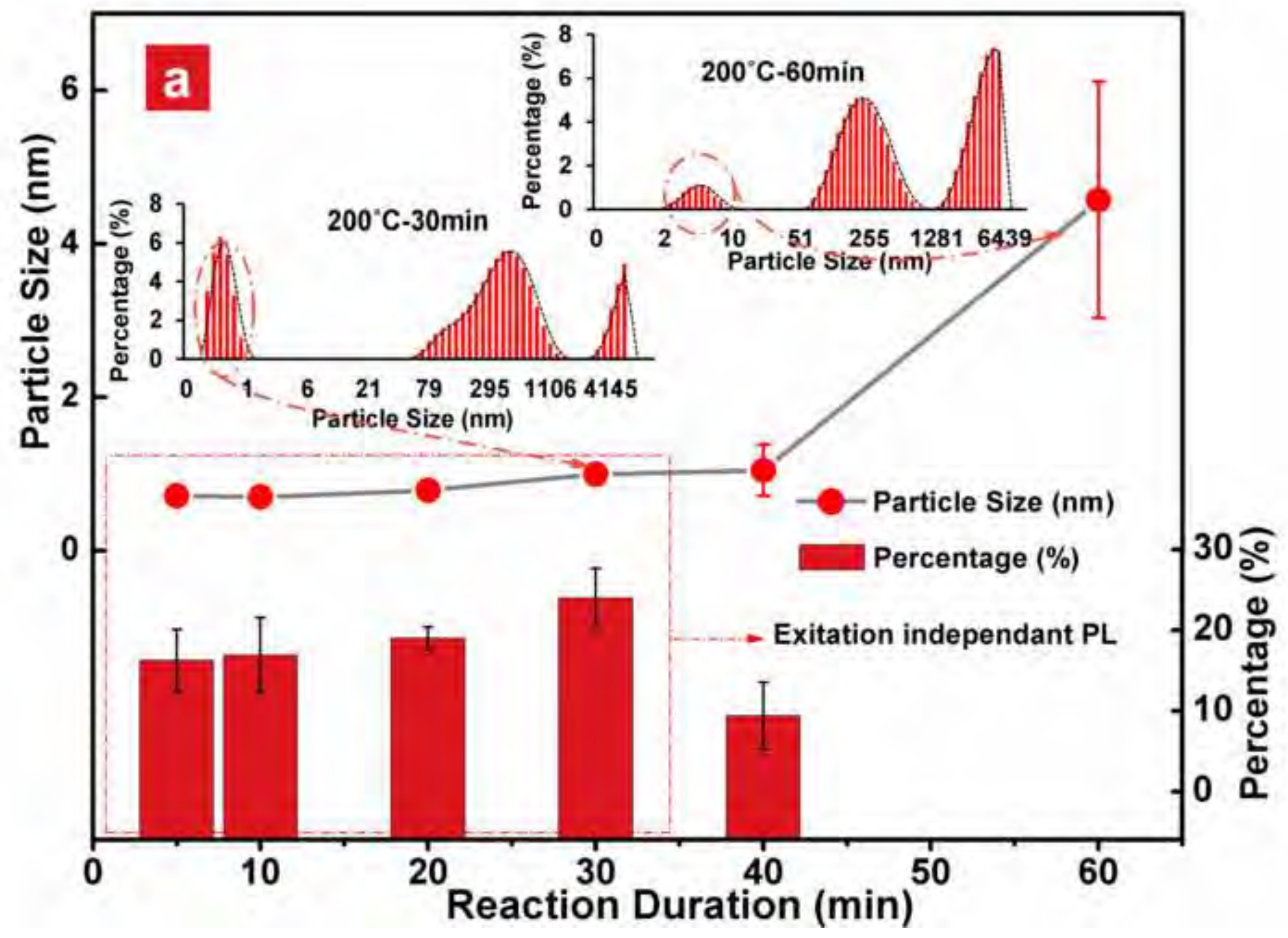




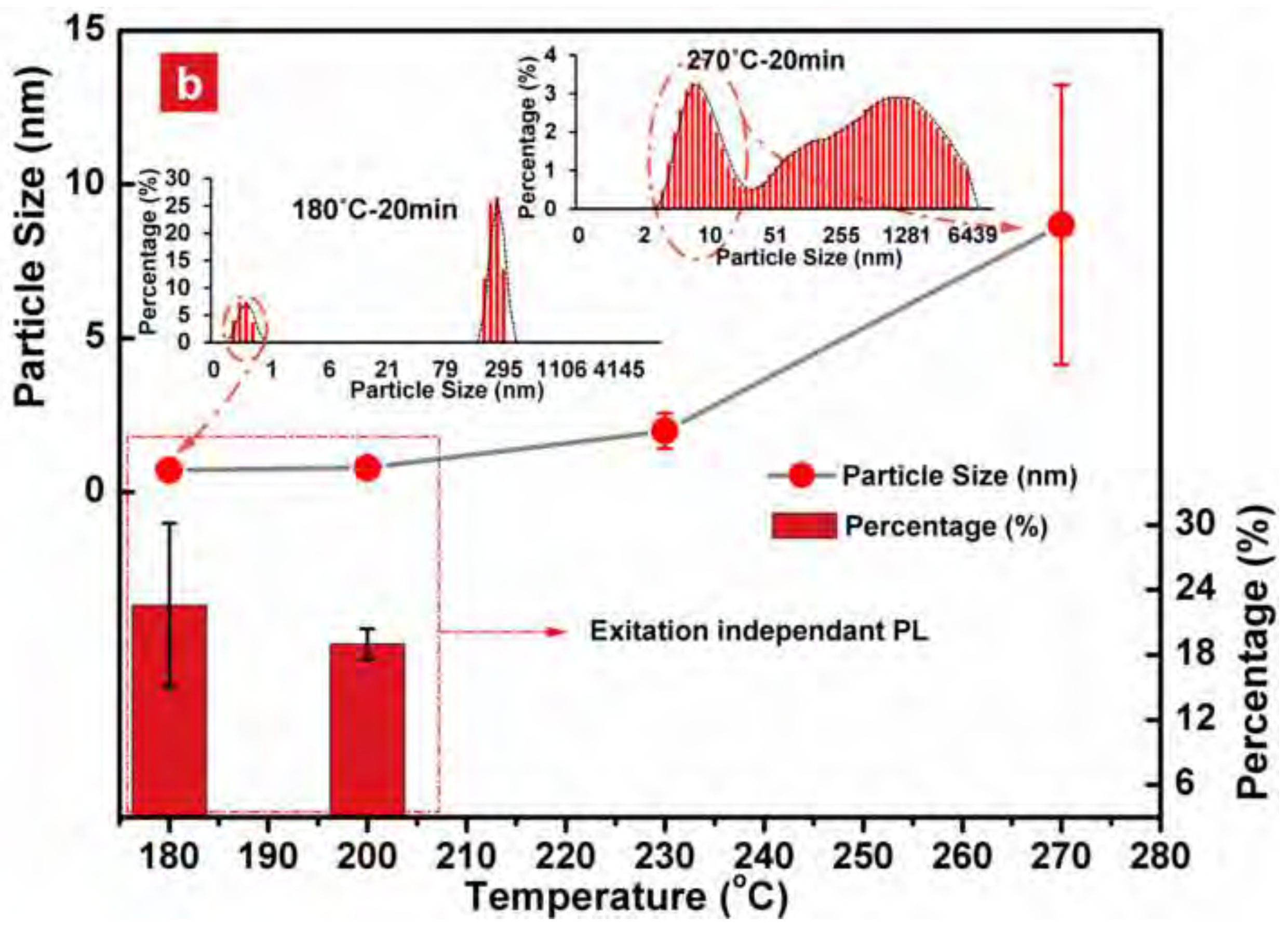




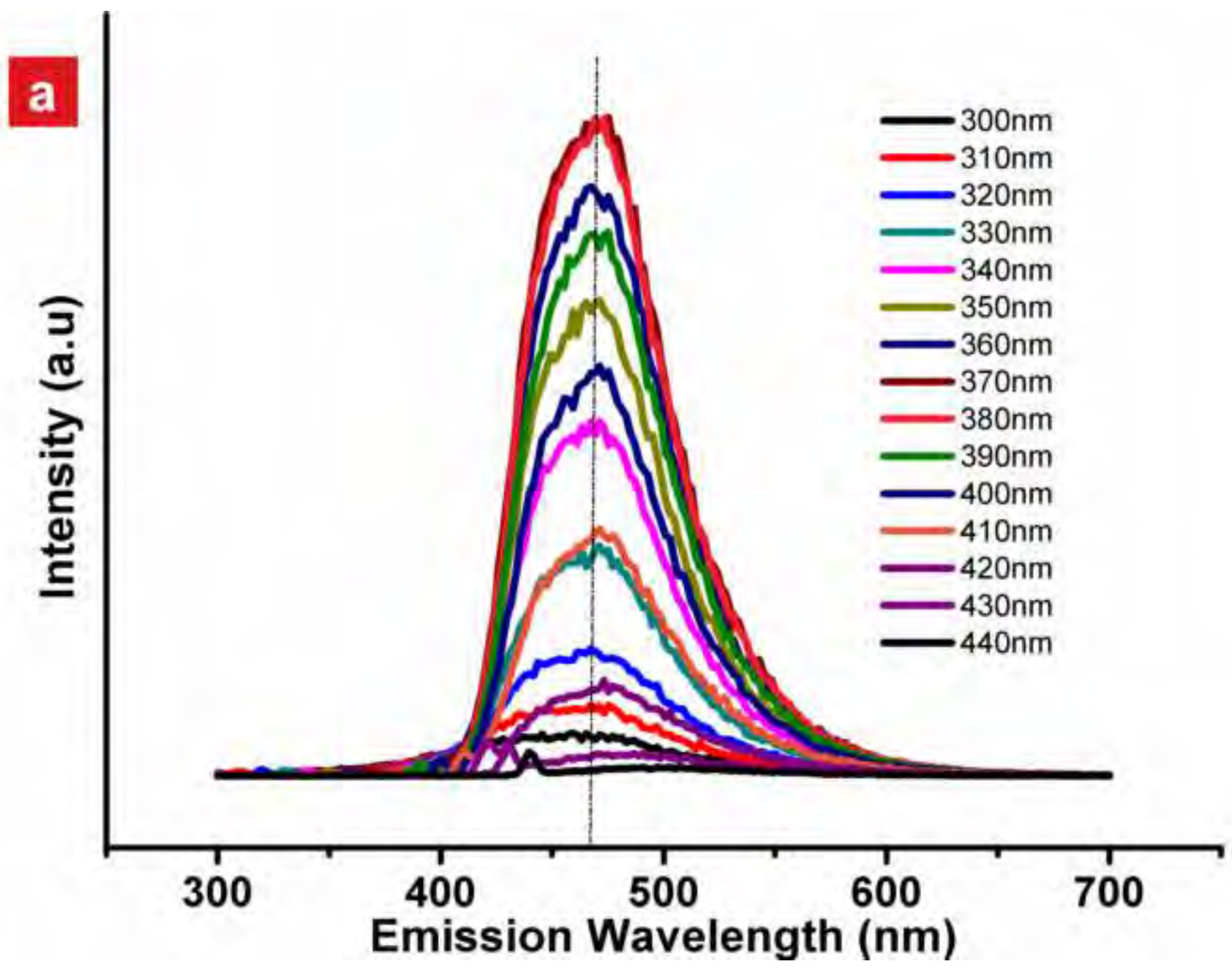




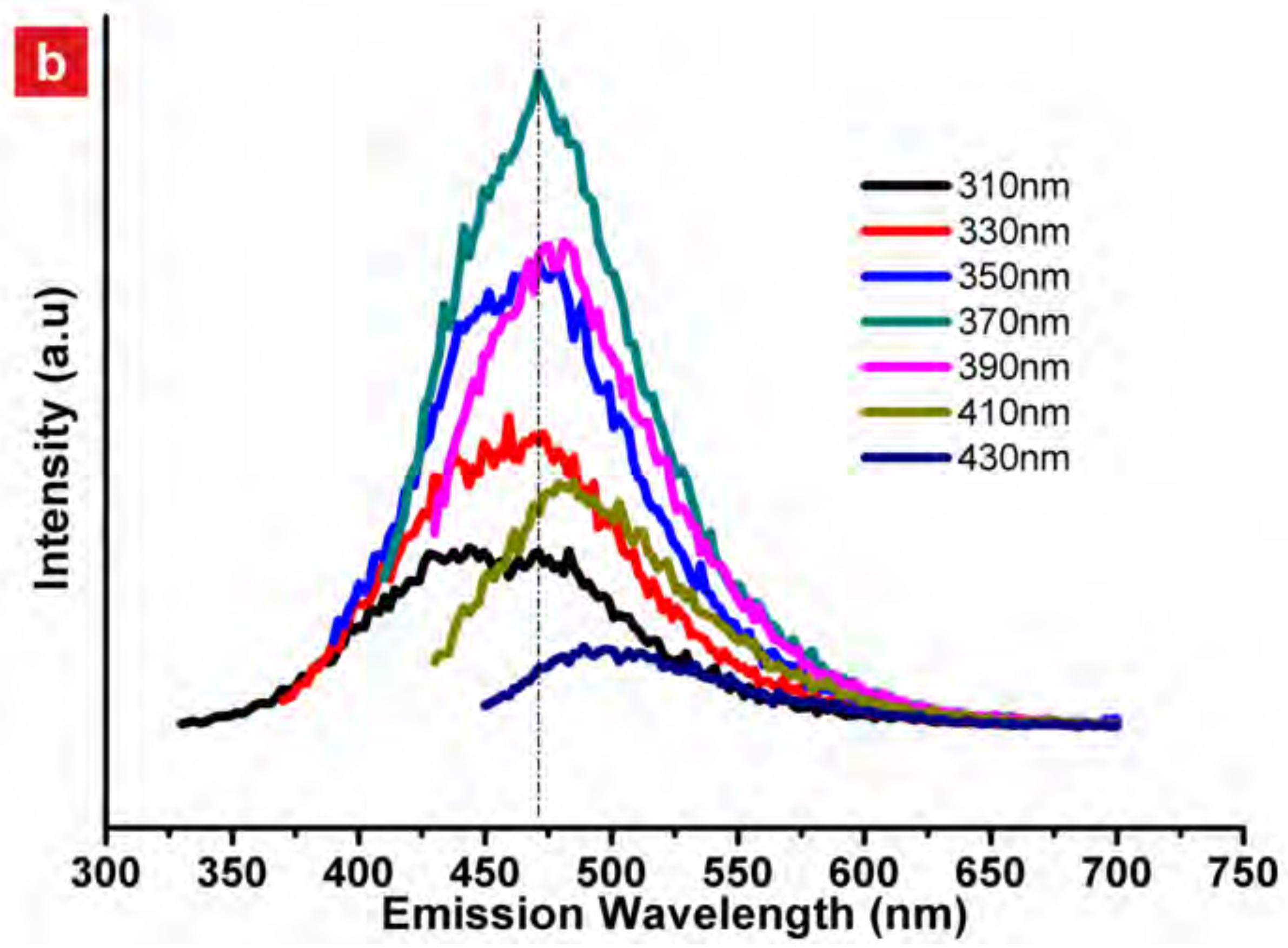




\section{$-\mathrm{COOH}$}
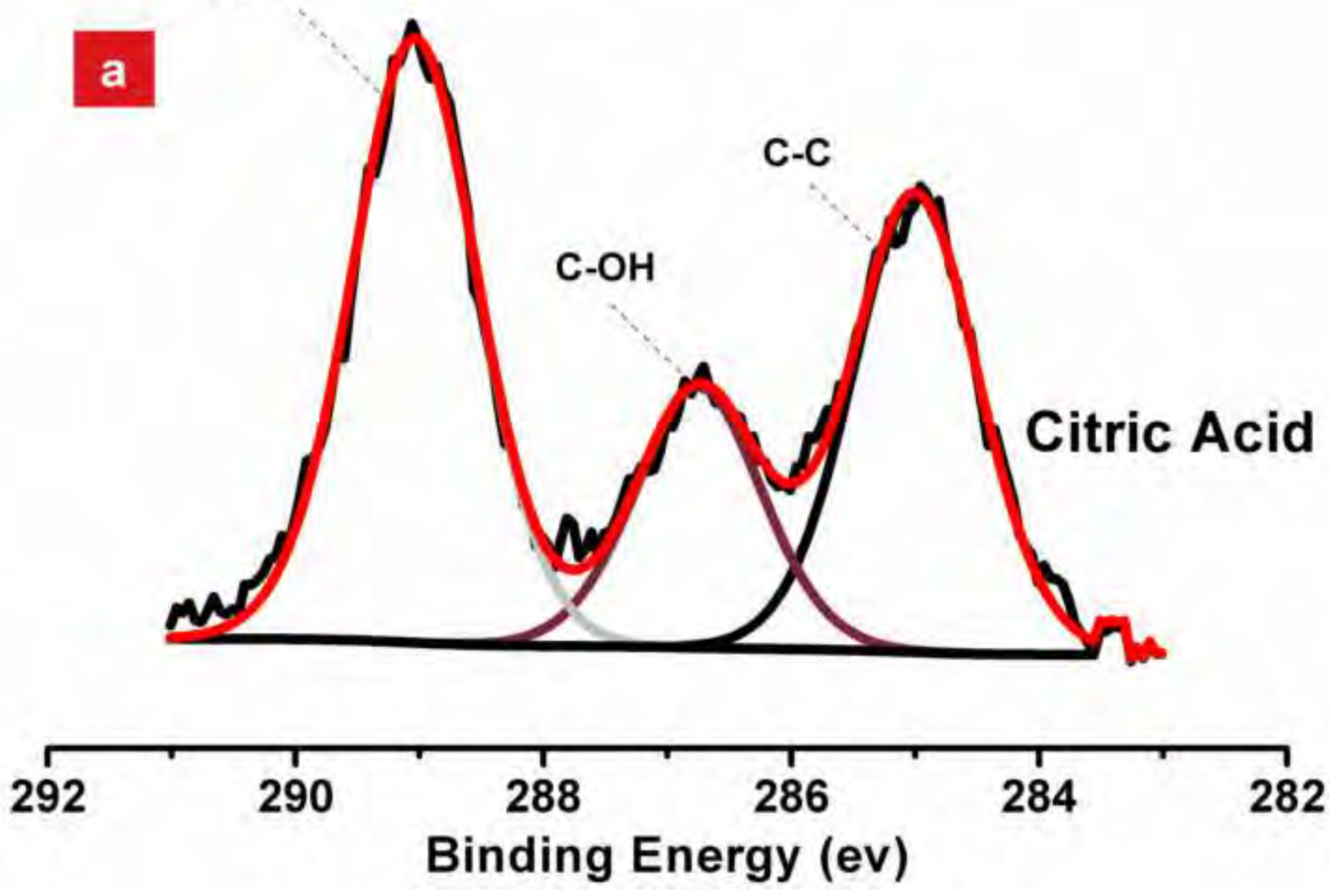


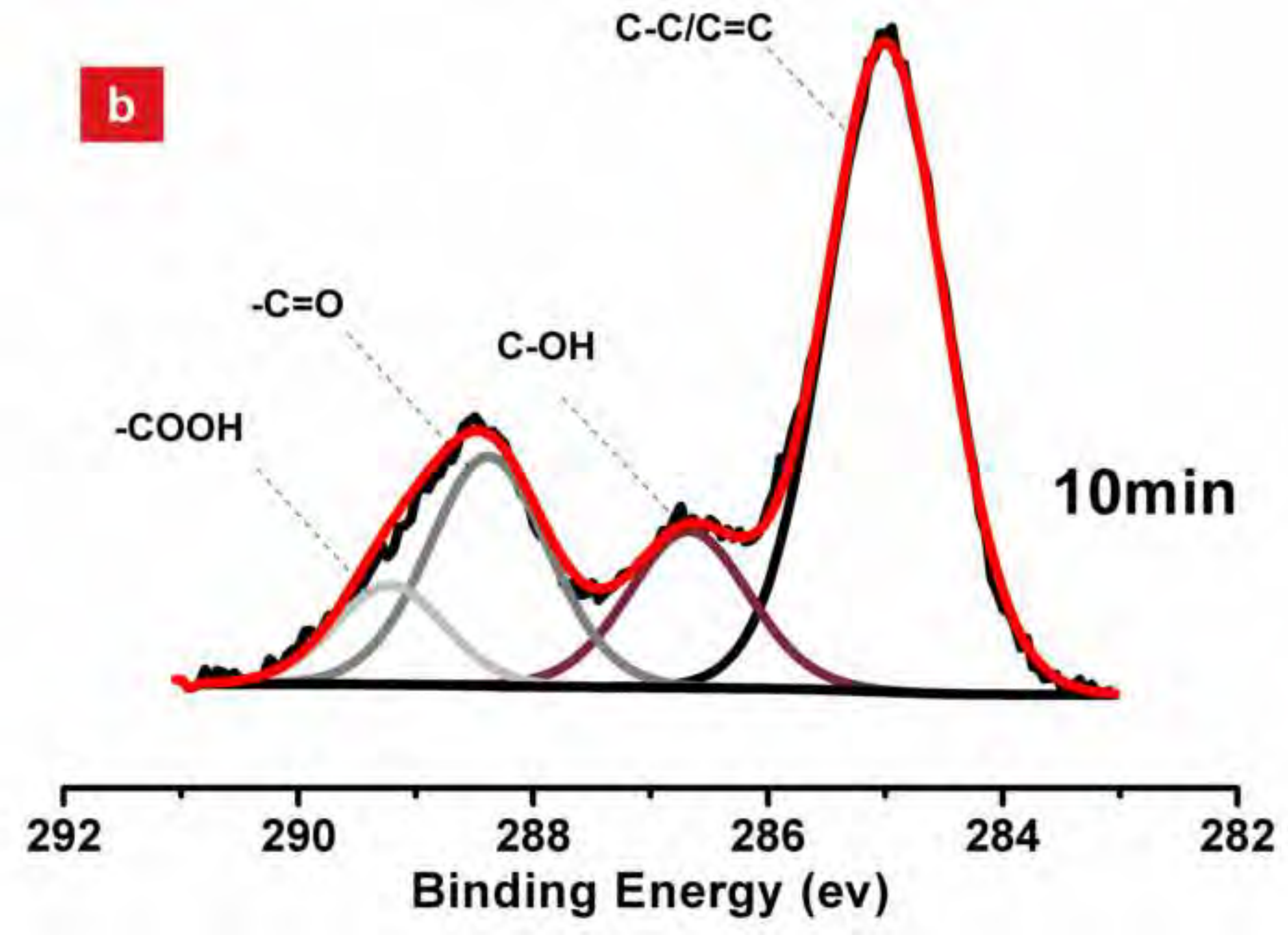




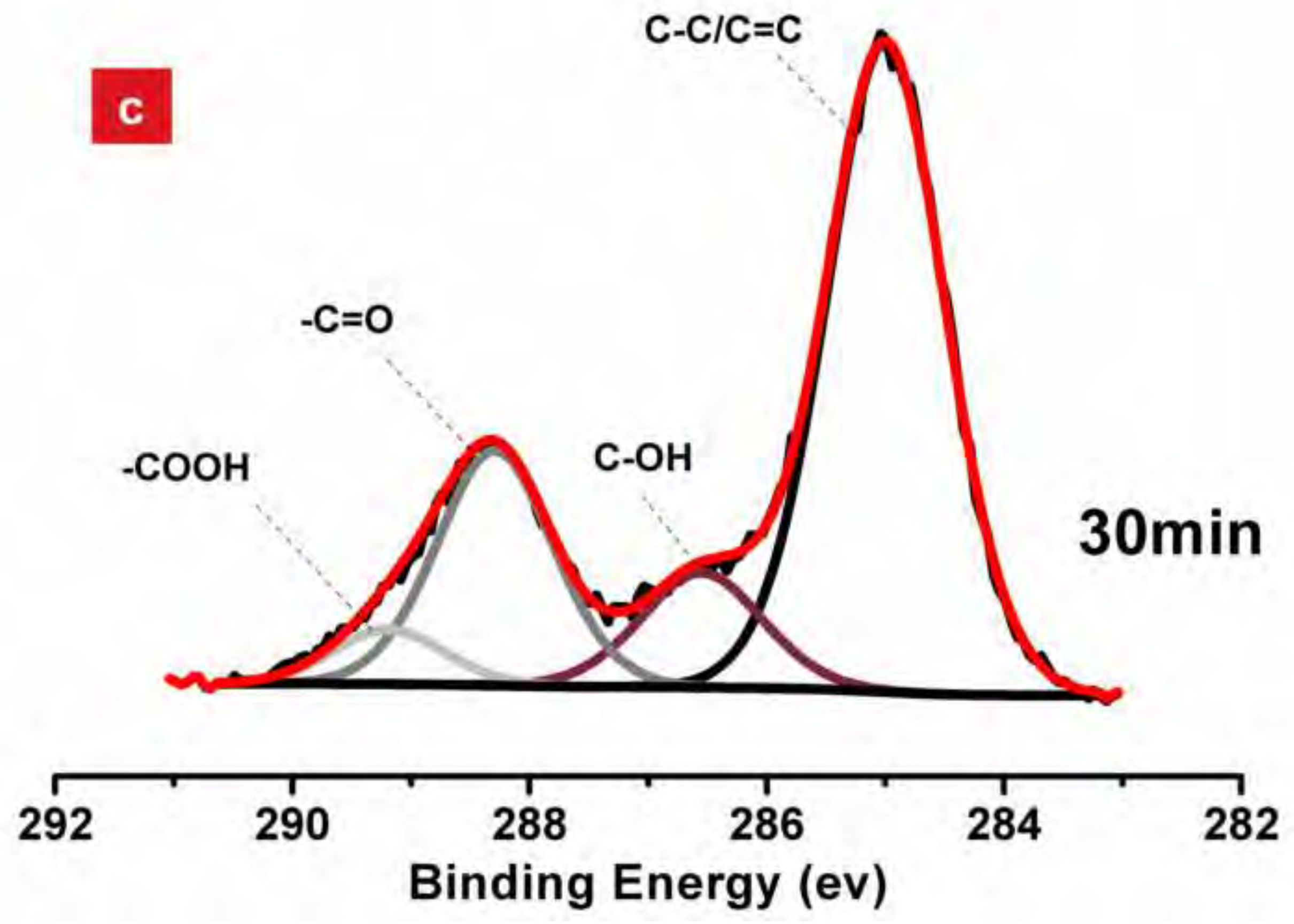



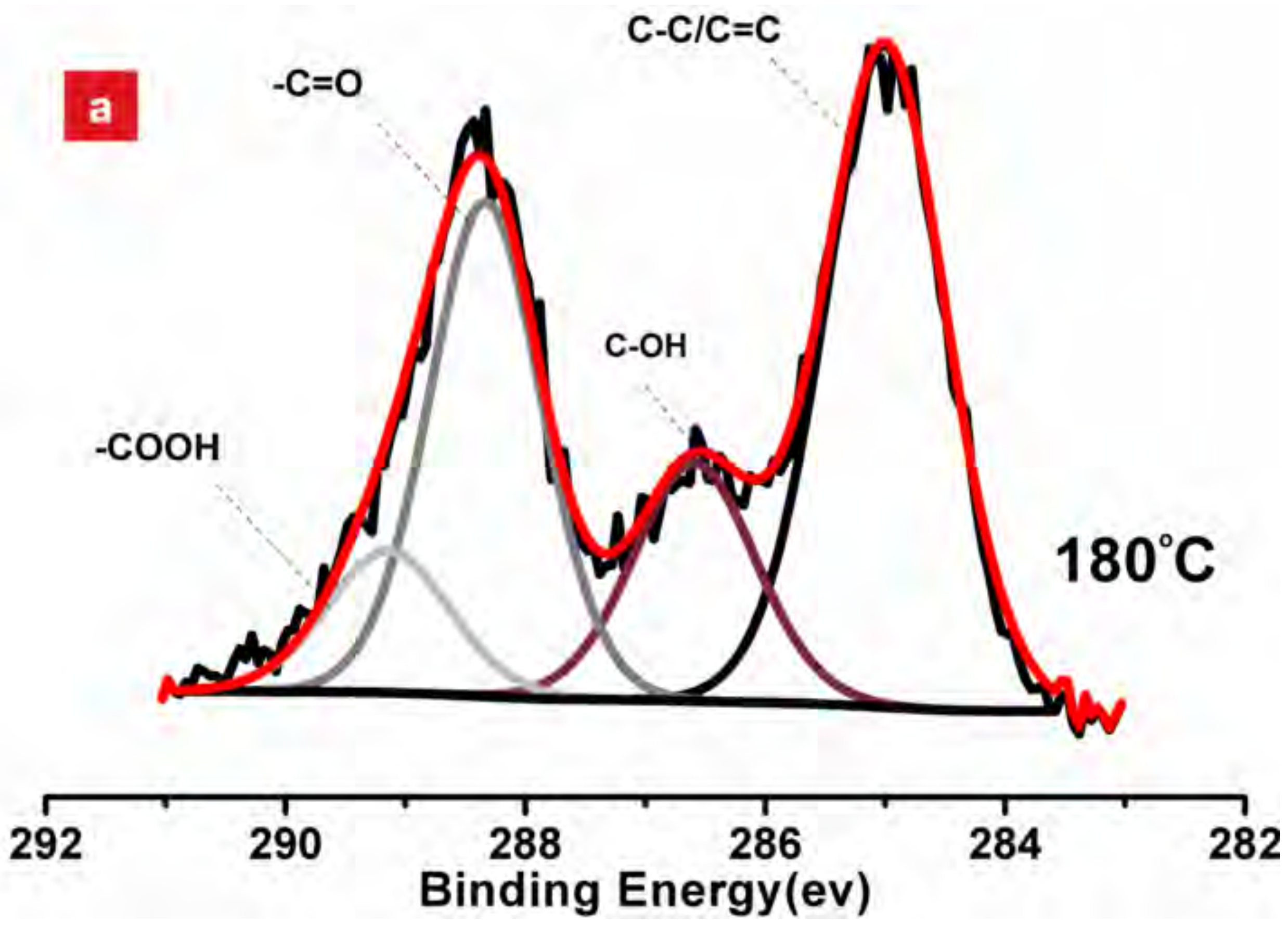


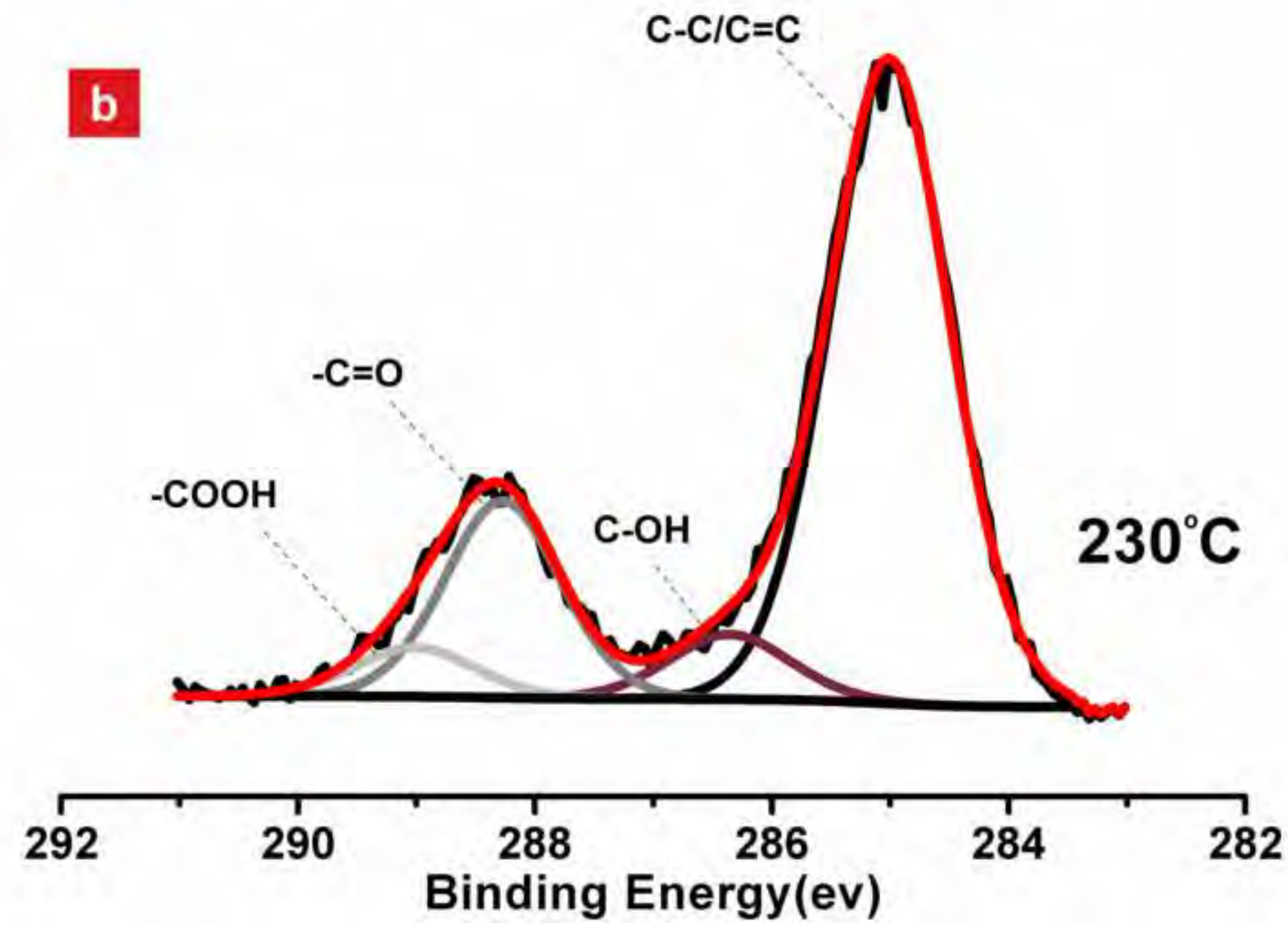




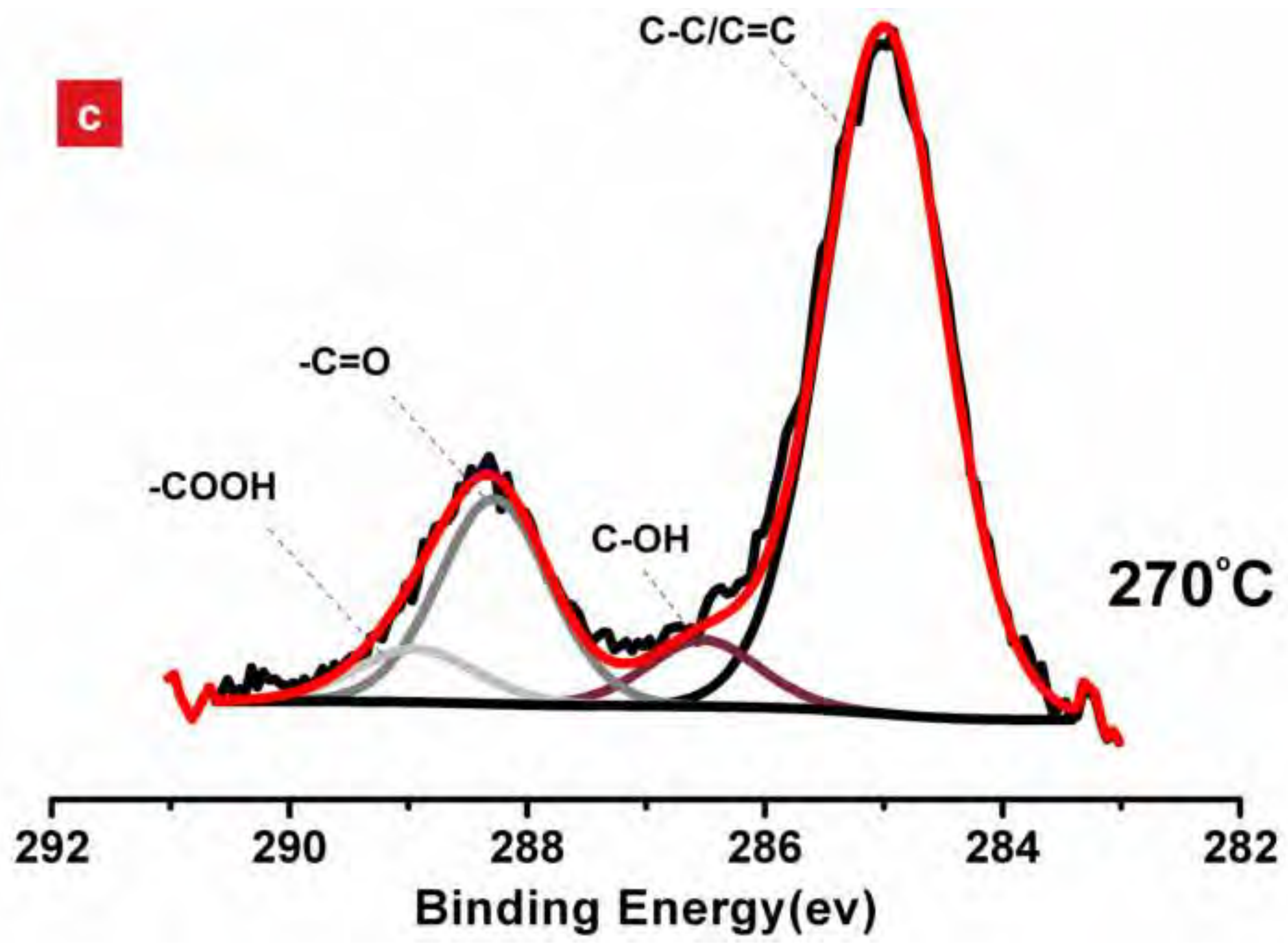


Click here to download high resolution image

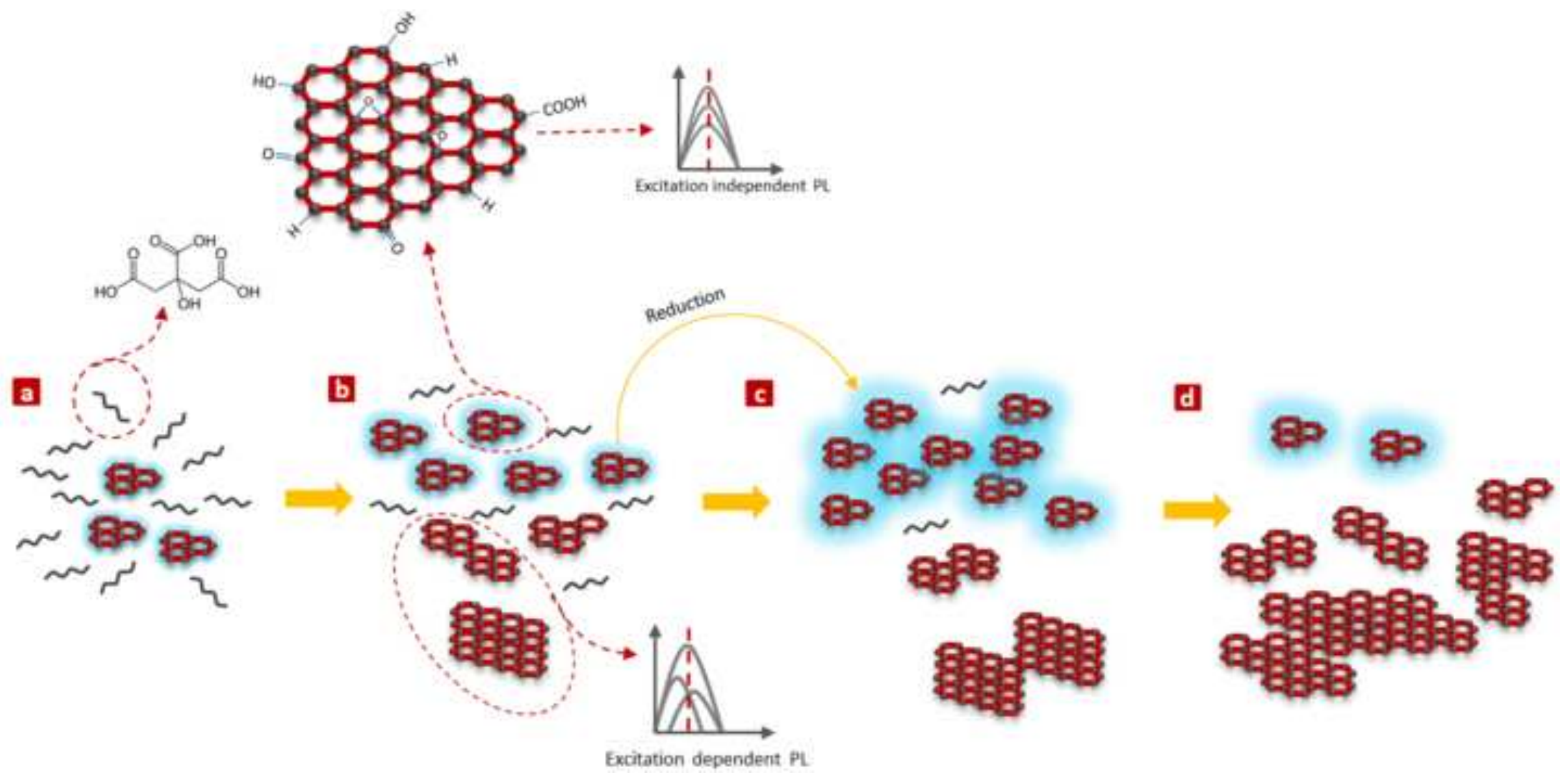




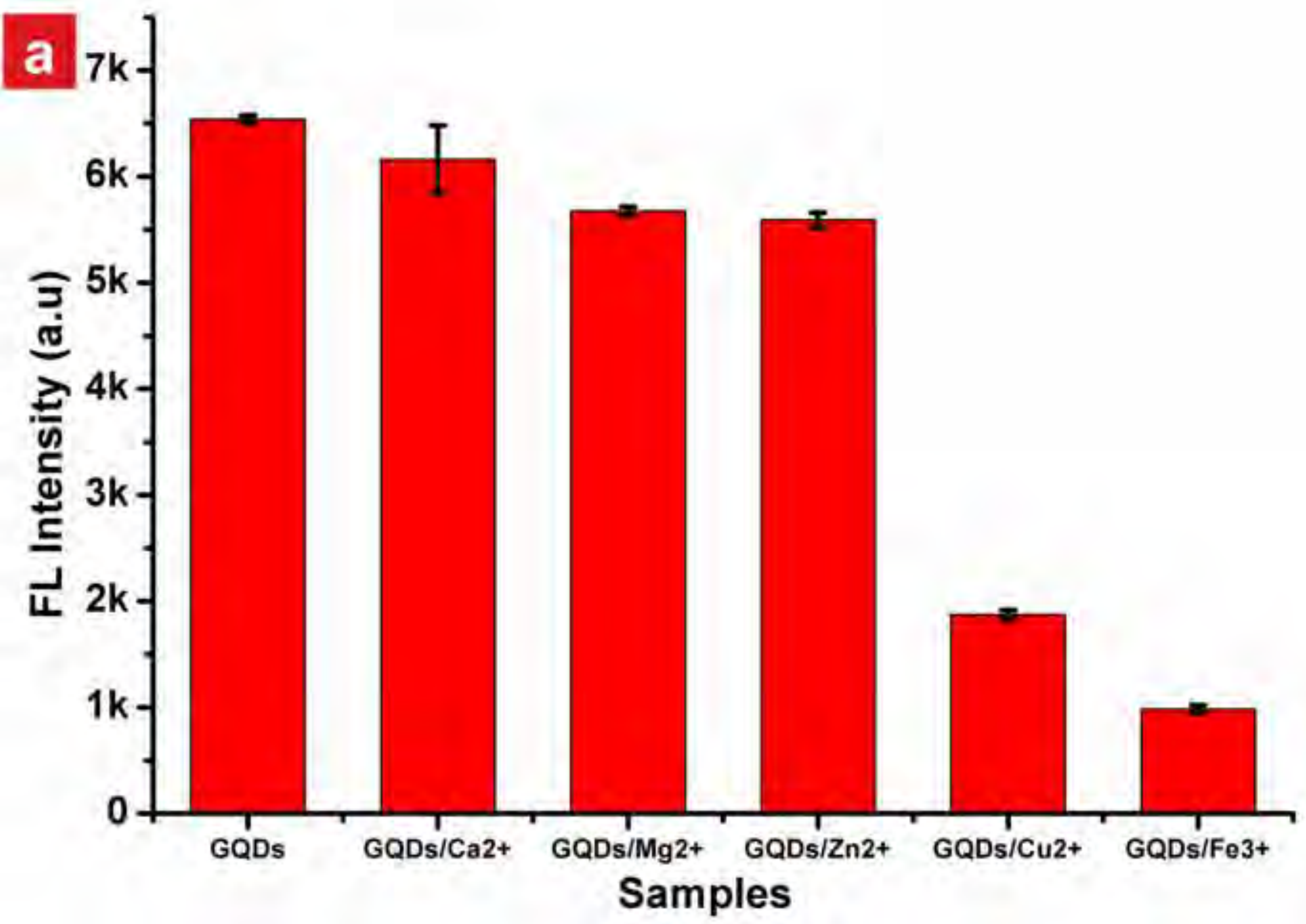




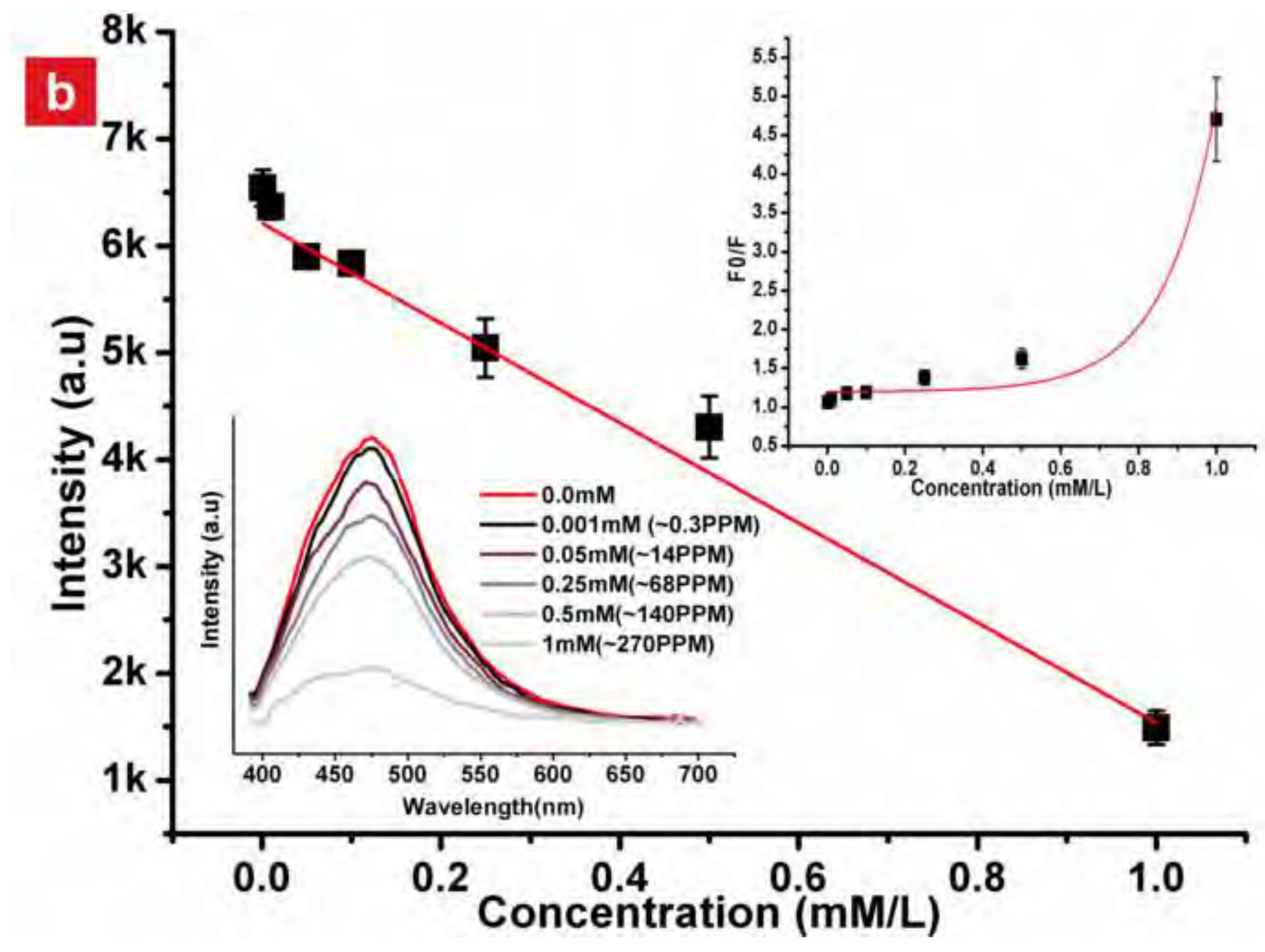


Click here to download high resolution image

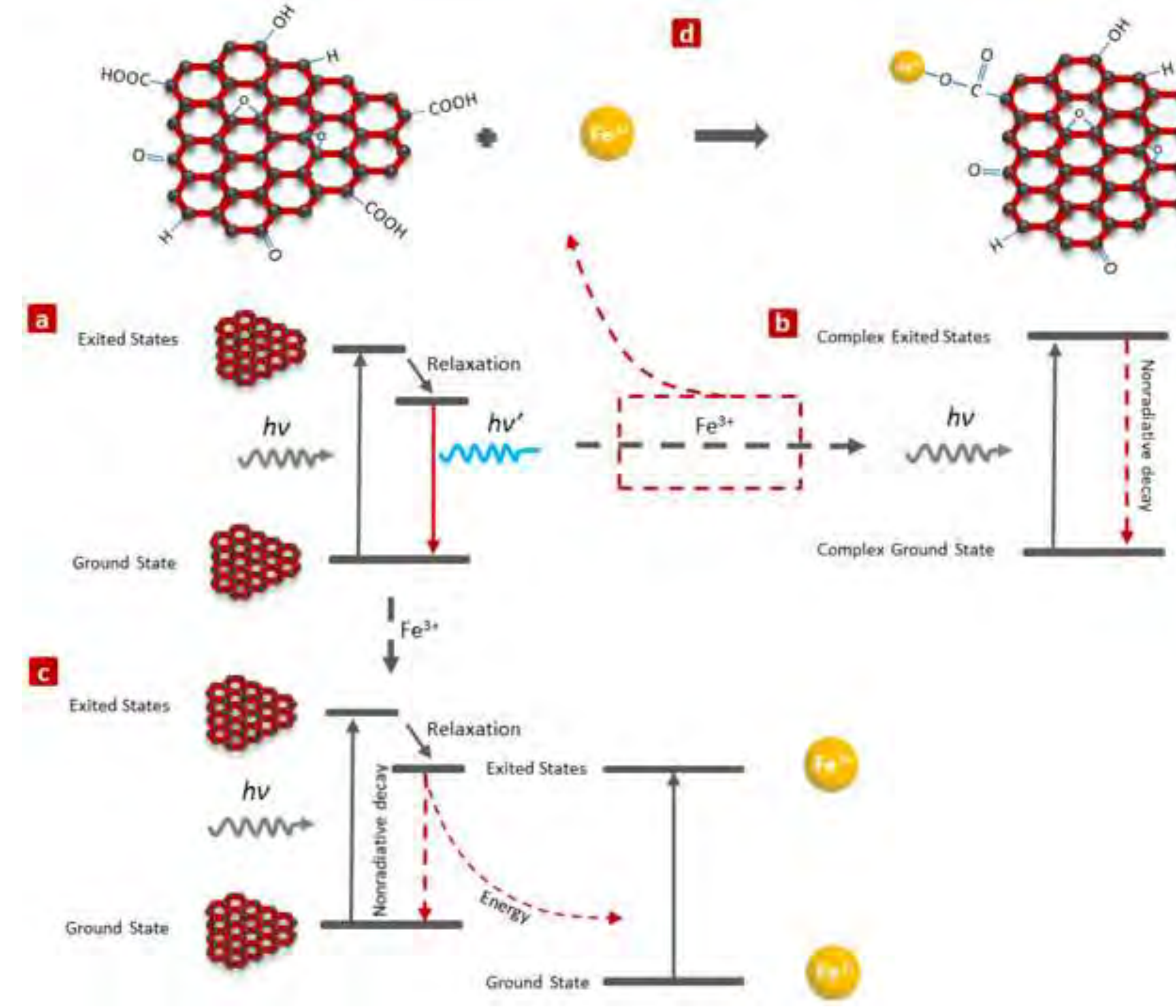

d 